\title{
Quasigeostrophic Turbulence with Explicit Surface Dynamics: Application to the Atmospheric Energy Spectrum
}

\author{
Ross Tulloch AND K. SHAFER SMITH \\ Center for Atmosphere Ocean Science, Courant Institute of Mathematical Sciences, New York University, New York, New York
}

(Manuscript received 16 October 2007, in final form 6 August 2008)

\begin{abstract}
The horizontal wavenumber spectra of wind and temperature near the tropopause have a steep -3 slope at synoptic scales and a shallower $-5 / 3$ slope at mesoscales, with a transition between the two regimes at a wavelength of about $450 \mathrm{~km}$. Here it is demonstrated that a quasigeostrophic model driven by baroclinic instability exhibits such a transition near its upper boundary (analogous to the tropopause) when surface temperature advection at that boundary is properly resolved and forced. To accurately represent surface advection at the upper and lower boundaries, the vertical structure of the model streamfunction is decomposed into four parts, representing the interior flow with the first two neutral modes, and each surface with its Green's function solution, resulting in a system with four prognostic equations. Mean temperature gradients are applied at each surface, and a mean potential vorticity gradient consisting both of $\beta$ and vertical shear is applied in the interior. The system exhibits three fundamental types of baroclinic instability: interactions between the upper and lower surfaces (Eady type), interactions between one surface and the interior (Charney type), and interactions between the barotropic and baroclinic interior modes (Phillips type). The turbulent steady states that result from each of these instabilities are distinct, and those of the former two types yield shallow kinetic energy spectra at small scales along those boundaries where mean temperature gradients are present. When both mean interior and surface gradients are present, the surface spectrum reflects a superposition of the interior-dominated -3 slope cascade at large scales, and the surface-dominated $-5 / 3$ slope cascade at small scales. The transition wavenumber depends linearly on the ratio of the interior potential vorticity gradient to the surface temperature gradient, and scales with the inverse of the deformation scale when $\beta=0$.
\end{abstract}

\section{Introduction}

The horizontal kinetic energy and potential temperature variance spectra near the tropopause, observed during the Global Atmospheric Sampling Program (GASP) and documented in Nastrom and Gage (1985, hereafter NG85), exhibit a shallow plateau at the largest wavelengths $(10000-3000 \mathrm{~km})$, a steep -3 spectral slope on synoptic scales $(\approx 3000-1000 \mathrm{~km})$, followed by a smooth transition (at a wavelength of about $450 \mathrm{~km}$ ) to a shallow $-5 / 3$ spectral slope on the mesoscales $(\approx 200-10 \mathrm{~km})$. The large and synoptic scale parts of the spectra are consistent with stirring by baroclinic instability near the Rossby deformation wavelength, feeding a forward cascade of enstrophy with a -3 slope,

Corresponding author address: K. Shafer Smith, Courant Institute of Mathematical Sciences, New York University, 251 Mercer St., New York, NY 10012.

E-mail: shafer@cims.nyu.edu as predicted by Charney's theory of geostrophic turbulence (Charney 1971). The mesoscale shallowing, however, does not fit easily into this picture; the robustness of the synoptic-scale slope and its consistency with geostrophic turbulence theory make the mesoscale spectral slope difficult to explain.

The key figure from NG85, reproduced in Fig. 1, plots power density spectra of the zonal wind $\left(u^{2}\right)$, meridional wind $\left(v^{2}\right)$, and the potential temperature $\left(\theta^{2}\right)$ as functions of horizontal wavenumber (meridional wind and potential temperature are offset by one and two orders of magnitude, respectively). Below wavelengths of about $5000 \mathrm{~km}$, the zonal and meridional wind spectra are nearly identical, implying isotropic kinetic energy [KE; equal to $\left(u^{2}+v^{2}\right) / 2$ ] in the synoptic scales and below. The potential temperature spectrum exhibits the same spectral slopes and spectral break, but is about half the magnitude of the spectra of the winds. The available potential energy (APE), equal to $g^{2} /\left(2 N \theta_{0}\right)^{2} \theta^{2}$, where $g$ is gravity, $N$ is the buoyancy frequency, and 


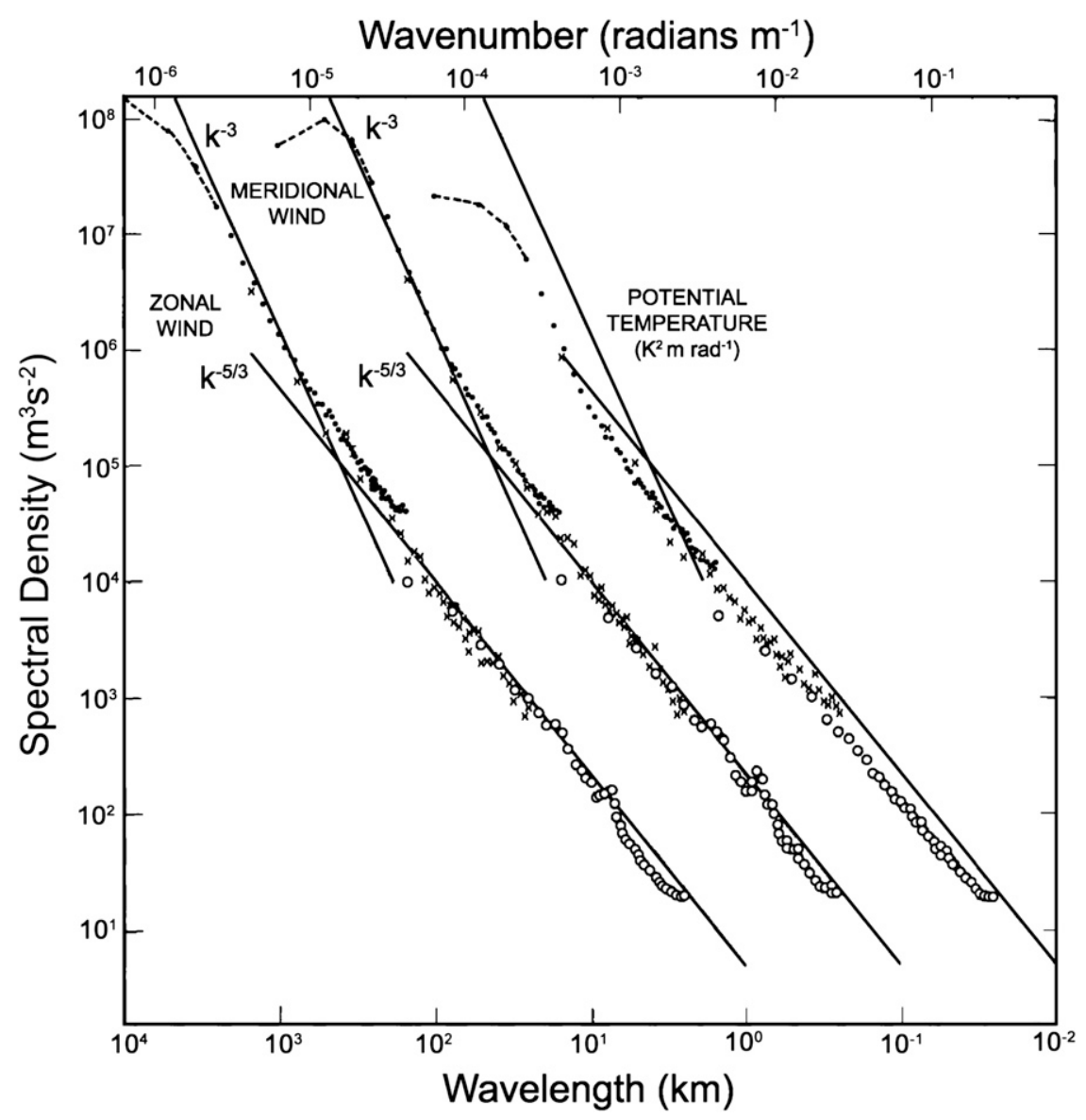

FIG. 1. Variance power spectra of wind and potential temperature near the tropopause from GASP aircraft data. The spectra for meridional wind and temperature are shifted one and two decades to the right, respectively; lines with slopes -3 and $-5 / 3$ are entered at the same relative coordinates for each variable for comparison. Reproduced with permission from Nastrom and Gage (1985).

$\theta_{0}$ is a reference potential temperature, is therefore very nearly in equipartition with the kinetic energy. ${ }^{1}$ In the -3 range, equipartition between KE and APE at each scale is also consistent with the predictions of geostrophic turbulence. ${ }^{2}$ High-resolution general circulation and weather forecasting models yield similar results, with equipartition in the -3 range, and a shal-

\footnotetext{
${ }^{1}$ Using typical upper-tropospheric values $\theta_{0}=300 \mathrm{~K}, N=10^{-2}$ $\mathrm{s}^{-1}$, and $g=9.8 \mathrm{~m} \mathrm{~s}^{-2}$, APE $\sim 2 \theta^{2}$, and so the dimensional prefactor just accounts for the drop in magnitude of the potential temperature relative to the winds.

${ }^{2}$ In quasigeostrophic theory, KE/APE $\sim\left(N h_{e} / f \ell_{e}\right)^{2}$, where $\ell_{e}$ and $h_{e}$ are the (variable) horizontal and vertical scales, respectively, of the eddying motion. Charney argued that, neglecting the influence of boundaries or inhomogeneities in the stratification, the forward cascade of potential enstrophy should be isotropic in the space $(x, y, N z / f)$. Thus, in the forward cascade, each scale of eddy motion will maintain a constant aspect ratio $\ell_{e} / h_{e} \sim N / f$, independent of scale, so KE/APE $\sim 1$.
}

lowing to $-5 / 3$ in the mesoscales (Koshyk and Hamilton 2001; Skamarock 2004; Takahashi et al. 2006; Hamilton et al. 2008).

Numerous theories for the mesoscale spectrum have been suggested over the past two decades (Lilly 1989; Vallis et al. 1997; Dewan 1979; VanZandt 1982; Koshyk et al. 1999; Tung and Orlando 2003a; Lindborg 2005; Kitamura and Matsuda 2006). Tung and Orlando (2003a) summarize all these theories, and put forward their own. In a recent paper, Tulloch and Smith (2006) also revisit the proposed mechanisms for the mesoscale shallowing, and propose a new one, based on a "toy" model that captures many aspects of the observations. The model is motivated by the following observations: (i) the near universality of the spectrum in the midlatitude atmosphere implies that synoptic-scale baroclinic instability is likely the forcing of both the -3 and $-5 / 3$ ranges of the spectrum (Tung and Orlando 2003a) and (ii) the $-5 / 3$ part of the spectrum is due to a forward 
energy cascade (Cho and Lindborg 2001; Tung and Orlando 2003b). The physical idea proposed is that advection of temperature perturbations at the tropopause leads to a forward cascade of temperature variance, resulting in $-5 / 3$ kinetic and potential energy spectra at mesoscales, as predicted by surface quasigeostrophic theory (SQG; Blumen 1978; Held et al. 1995). This is rationalized by the analysis of Juckes (1994), who showed that tropopause temperature anomalies account for about $80 \%$ of the tropospheric eddy field. The novel aspect of Tulloch and Smith (2006) was the demonstration that by limiting surface quasigeostrophic flow to a finite depth, a natural transition scale emerges, and when the flow is forced at large scale, the kinetic energy spectrum slope is -3 between the forcing and transition scales, and $-5 / 3$ below the transition scale. The transition scale $(\sim N H / f$, where $N$ is the buoyancy frequency, $f$ is the Coriolis parameter, and $H$ is the depth of the fluid-i.e., the shallow-water deformation scale) is the scale above which the surface signals can "feel" the lower boundary. The large-scale limit is that of barotropic flow, and so follows the predictions of two-dimensional turbulence, while the small-scale limit, where the vertical extent of temperature signals is much less than $H$, is that of surface quasigeostrophic flow.

The finite-depth SQG model is compelling because it leads to a forward cascade of vortical energy, consistent with observations (Cho and Lindborg 2001; Cho et al. 1999), and the cascade can be generated by large-scale forcing such as baroclinic instability, ubiquitous throughout the midlatitudes, thereby accounting for the universality of the spectra (Nastrom and Gage 1985). Taken as complete, however, the model has the following deficiencies: (i) it fails to explain the available potential energy spectrum at synoptic scales (finite-depth surface quasigeostrophic flow has no significant potential energy at large scales); (ii) it predicts a transition scale that is larger than that observed; and (iii) it predicts an evanescent spectrum at small scales when moving down into the troposphere (midlevel spectral observations in the troposphere are rare, but there is no evidence for evanescent decay of energy). These deficiencies, it is shown here, are removed when interior potential vorticity anomalies are included in the flow.

The surface quasigeostrophic model is derived by assuming quasigeostrophic scaling, and considering a special case in which temperature on a horizontal surface is advected and bounded above and/or below by regions of zero or constant potential vorticity (PV). It is thus typically assumed that regions of constant PV are necessary in order to obtain dynamics like those predicted by surface quasigeostrophic flow. In a companion paper to this one (Tulloch and Smith 2009), we show that this restriction is not necessary, and that surface effects of the type predicted by SQG occur whenever temperature perturbations are forced and sufficiently resolved in the vertical (see also McWilliams and Chow 1981; Fox-Rabinovitz and Lindzen 1993; Solomon and Lindzen 2000; Snyder et al. 2003).

In the present paper, we show that surface effects can be retained without high-vertical resolution, so long as representations of the surface dynamics are included explicitly in the model. The method proposed follows recent work by Lapeyre and Klein (2006), but goes back to Hoskins et al. (1985) and perhaps earlier papers (see also, e.g., Holopainen and Kaurola 1991; Davies and Bishop 1994). The method is based on the decomposition of the streamfunction into components that solve the interior PV and two boundary temperature problems, separately; it is an equivalent alternative to Bretherton's (1966) use of delta function layers of PV at rigid surfaces (and both methods can be understood in terms of a Green's function, as shown in appendix A). The decomposition method, however, has the advantages that it allows for the construction of a fully nonlinear numerical model that both captures the surface dynamics accurately (without need for high vertical resolution), and makes explicit their interaction with the interior flow. The Bretherton approach, by contrast, relies on finite-difference representations of the delta sheets of $\mathrm{PV}$, with the accompanying pitfalls described in the previous paragraph.

We specialize within this model framework to include just enough complexity to address the observed atmospheric energy spectra, at the transition to subsynoptic scales. The simplified model advects the barotropic and baroclinic interior flow, and the upper and lower surface temperatures. In spectral space, the vertical structures for the four variables are separable from their timedependent parts. Assuming constant stratification $N^{2}$, these modes can be easily computed analytically. The resulting model is very similar to one developed by G. Flierl (2007, personal communication), with some differences, but to our knowledge these are the only examples of fully nonlinear, forward model implementations using the decomposition approach. This two-mode, two-surface model (hereafter referred to as the TMTS model) is effectively a hybrid of the Phillips and Eady models, and so can represent baroclinic instability generated from barotropic-baroclinic interactions (as in the standard two-layer Phillips model), surface-surface interactions (as in the Eady model), and from interactions between either surface and the interior (as in the Charney model of baroclinic instability). It is hoped that, besides its application in this paper, the analytical tractability of this four-variable 
model may allow for its use as a pedagogical tool (we thank an anonymous reviewer for suggesting this).

The TMTS model is designed to understand the observed atmospheric energy spectrum at subsynoptic scales; a corollary is that high vertical resolution is not needed to understand the transition to a shallow spectrum, so long as surface dynamics are explicitly represented. On the other hand, the model may be deficient for applications that require a more realistic representation of the vertical structure of eddy fluxes at the synoptic and planetary scale.

Both linear instabilities and nonlinear turbulent steady states of the TMTS model are explored. Numerical simulations of turbulent steady states reveal spectra similar to the observed atmospheric spectra in Fig. 1, and can be understood as resulting from the superposition of a -3 slope, due to interior-forced geostrophic turbulence at large scales, and a $-5 / 3$ slope, due to surface-forced SQG turbulence at small scales. This structure is in accordance with Fig. 7 from Lindborg (1999) which shows that the NG85 spectrum is well fit by a superposition. Rather than resulting from surface signals feeling the bottom boundary, the transition here occurs where the surface cascade starts to dominate the interior cascade. The scale at which the transition occurs, it is shown, is a function of the ratio of interior PV to surface temperature gradients. The transition scale predicted by applying the formula to the National Centers for Environmental Prediction (NCEP) reanalysis data is very close to the observed transition scale.

In summary, this paper is presented with the following major goals in mind:

1) to show the usefulness of the streamfunction-decomposition method in constructing numerical models that accurately represent surface dynamics;

2) to derive a simplified model (TMTS) within this framework that captures all major types of baroclinic instability;

3) to use this model to demonstrate that surface dynamics at the tropopause may explain the transition to a shallow energy spectrum at subsynoptic scales; and

4) to provide a theory for the transition scale observed in the atmospheric energy spectra.

The paper is organized as follows: In section 2 we review the quasigeostrophic equations, including advection by mean wind and the appropriate boundary conditions, and derive a simplified model consisting of two interior and two surface modes (i.e., the TMTS model). Solutions to the associated linear instability problem are presented in section 3, partly in order to characterize the parameter space of interest, but also to demonstrate the ability of the model to represent the basic types of baroclinic instability. The results of a series of nonlinear simulations are presented in section 4 , and a theory for the transition scale that is consistent with both simulated and observed data is proposed. Successes and shortcomings of the theory are discussed in section 5, along with plans for future work.

\section{A quasigeostrophic model for surface-interior interaction}

For the sake of providing a self-contained presentation, the quasigeostrophic equations are stated and the two-internal, two-surface mode approximation is derived as a stand-alone model. A more complete treatment is discussed briefly in Tulloch and Smith (2009), and implications for more complex interior structure (representative of the ocean) will be explored in a future paper.

\section{a. The quasigeostrophic equations}

The quasigeostrophic equations for a fluid bounded in the vertical by flat, rigid surfaces at $z=H$ and $z=0$, and assuming a mean baroclinic zonal wind $U(z)$, are

$$
\begin{aligned}
& q_{t}+J(\psi, q)+U q_{x}+v Q_{y}=0 \text { for } 0<z<H, \\
& \theta_{t}+J(\psi, \theta)+U \theta_{x}+v \Theta_{y}=0 \text { for } z=H, \quad \text { and } \\
& \theta_{t}+J(\psi, \theta)+U \theta_{x}+v \Theta_{y}=-r \nabla^{2} \psi \text { for } z=0
\end{aligned}
$$

where $\psi(x, y, z, t)$ is the geostrophic streamfunction, $\mathbf{u}=(u, v)=\left(-\psi_{y}, \psi_{x}\right)$ is the horizontal velocity, $\nabla=$ $\left(\partial_{x}, \partial_{y}\right), J(A, B)=A_{x} B_{y}-A_{y} B_{x}$ is the two-dimensional Jacobian operator, and $r=\delta_{\mathrm{Ek}} N^{2} / f$ is the (linear) Ekman drag coefficient, where $\delta_{\mathrm{Ek}}$ is proportional to the thickness of the Ekman layer, $N$ is the buoyancy frequency, and $f$ is the Coriolis parameter. The potential vorticity $q$ and potential temperature $\theta$ are related to the streamfunction by

$$
q=\nabla^{2} \psi+\Gamma \psi \quad \text { and }\left.\quad \theta\right|_{z=0, H}=\left.\psi_{z}\right|_{z=0, H}
$$

respectively, where $\Gamma \equiv \partial_{z} f^{2} / N^{2} \partial_{z}$ is the stretching operator. The temperature variable $\theta$ is scaled by $g / f \theta_{0}$, where $g$ is gravitational acceleration and $\theta_{0}$ is a reference temperature, and so the advected "temperatures," as well as the linear drag coefficient $r$, have the units of velocity. Note also that in an atmospheric context, $z$ is a pseudoheight, or modified pressure coordinate (Hoskins and Bretherton 1972). The mean gradients of potential vorticity and potential temperature are related to the mean wind and Coriolis gradient $\beta$ by 
$Q_{y}=\beta-\Gamma U, \Theta_{y}(0)=-U_{z}(0), \Theta_{y}(H)=-U_{z}(H)$.

Since the potential vorticity inversion problem in (2) is linear we can decompose the total streamfunction into three components:

$$
\psi=\psi^{I}+\psi^{T}+\psi^{B}
$$

where each of the above depends on $(x, y, z, t)$, the interior (I) part $\psi^{I}$ solves

$$
\nabla^{2} \psi^{I}+\Gamma \psi^{I}=q,\left.\quad \psi_{z}^{I}\right|_{z=H}=0,\left.\quad \psi_{z}^{I}\right|_{z=0}=0,
$$

and the top (T) and bottom (B) surface components $\psi^{T}$ and $\psi^{B}$ solve

$$
\begin{gathered}
\nabla^{2} \psi^{T}+\Gamma \psi^{T}=0,\left.\quad \psi_{z}^{T}\right|_{z=H}=\left.\theta\right|_{z=H},\left.\quad \psi_{z}^{T}\right|_{z=0}=0, \quad \text { and } \\
\nabla^{2} \psi^{B}+\Gamma \psi^{B}=0,\left.\quad \psi_{z}^{B}\right|_{z=H}=0,\left.\quad \psi_{z}^{B}\right|_{z=0}=\left.\theta\right|_{z=0} .
\end{gathered}
$$

Similar decompositions have been used in the past; for example, Davies and Bishop (1994) applied such a decomposition to edge waves with interior PV distributions, and Lapeyre and Klein (2006) used it as framework through which to interpret oceanic surface signals. See appendix A for a comparison between this streamfunction decomposition and the method that considers the boundary temperature distribution as sheets of potential vorticity (Bretherton 1966; Heifetz et al. 2004).

\section{b. Modal representation}

To generate a numerical model that will aid understanding of the atmospheric energy spectrum, the following simplifying assumptions are made: (i) horizontal boundary conditions are taken to be periodic, consistent with the assumption of horizontal homogeneity in the synoptic scales and below; (ii) the vertical structure of the interior flow can be represented with the gravest two standard vertical modes (barotropic and baroclinic); (iii) the stratification $N^{2}$ is constant in the troposphere and infinite above, so that the tropopause itself is a rigid lid (this assumption can be relaxed, following Juckes 1994); and (iv) the mean velocity is zonal, horizontally constant and projects onto the baroclinic and surface modes (with no barotropic component).

Assumption (i) allows a Fourier representation in the horizontal, and so the full streamfunction can be written as

$$
\psi(x, y, z, t)=\sum_{\mathbf{K}} e^{i \mathbf{K} \cdot \mathbf{x}}\left[\hat{\psi}_{\mathbf{K}}^{I}(z, t)+\hat{\psi}_{\mathbf{K}}^{T}(z, t)+\hat{\psi}_{\mathbf{K}}^{B}(z, t)\right],
$$

where $\mathbf{K}=(k, \ell)$ is the horizontal wavenumber and $K=$ $|\mathbf{K}|$. Hats denote spectral coefficients, and from hereafter, where no confusion can arise the subscript $\mathbf{K}$ will be dropped.

Assumption (ii) allows for the expansion of the interior part in modes $\hat{\psi}^{I}(z, t)=\hat{\psi}^{\mathrm{bt}}(t)+\hat{\psi}^{\mathrm{bc}}(t) \phi(z)$, where, where bt means barotropic, bc means baroclinic, and $\phi(z)$ is the first baroclinic mode, that is, the gravest, nonconstant eigenfunction solution to

$$
\Gamma \phi=-\lambda^{2} \phi \quad \text { for } \quad \frac{d \phi}{d z}(0, H)=0 .
$$

It is straightforward, but cumbersome, to use more vertical modes in the interior. With assumption (iii), the mode is

$$
\phi(z)=\sqrt{2} \cos \left(\frac{\pi z}{H}\right) \quad \text { and } \quad \lambda=\frac{\pi f}{N H} .
$$

Note for future reference that $\langle\phi\rangle=\left\langle(\phi)^{3}\right\rangle=0$ and $\left\langle(\phi)^{2}\right\rangle=1$, where $\langle\cdot\rangle=H^{-1} \int_{0}^{H} \cdot d z$ is shorthand for a vertical average.

In the spectral domain, the surface inversion problems in (6a) and (6b) are separable, and so $\hat{\psi}^{T}(z, t)=\hat{\psi}^{t}(t) \phi^{t}(z)$ and $\hat{\psi}^{B}(z, t)=\hat{\psi}^{b}(t) \phi^{b}(z)$, where lowercase $t$ (top) and b (bottom) are used to distinguish the separated variables and $\phi^{t, b}$ (which we call the "surface modes") satisfy

$$
\begin{aligned}
& \left(-K^{2}+\Gamma\right) \phi^{t}=0, \quad \frac{d \phi^{t}}{d z}(0)=0, \quad \frac{d \phi^{t}}{d z}(H)=\frac{\hat{\theta}^{t}}{\hat{\psi}^{t}} \\
& \left(-K^{2}+\Gamma\right) \phi^{b}=0, \quad \frac{d \phi^{b}}{d z}(0)=\frac{\hat{\theta}^{b}}{\hat{\psi}^{b}}, \quad \frac{d \phi^{b}}{d z}(H)=0 .
\end{aligned}
$$

Unlike the interior modes, the surface modes will depend on the magnitude of the wavevector, $\mathbf{K}$. Note also that $\phi^{t}(H)=1$ and $\phi^{b}(0)=1$, so that $\hat{\psi}^{T}(H, t)=\hat{\psi}^{t}(t)$ and $\hat{\psi}^{B}(0, t)=\hat{\psi}^{b}(t)$. Since $N^{2}$ is constant, the solutions are easily computed; they are

$$
\begin{aligned}
\phi^{t}(z) & =\cosh \left(\mu \frac{z}{H}\right) \operatorname{sech} \mu, \\
\phi^{b}(z) & =\cosh \left(\mu \frac{z-H}{H}\right) \operatorname{sech} \mu,
\end{aligned}
$$

where $\mu=K N H / f$.

\section{c. Inversion relations}

Given the surface modes, the top and bottom temperature fields are obtained by setting $z=H$ in $\phi^{t}$ and $z=0$ in $\phi^{b}$, leading to

$$
\left.\hat{\theta}^{t}(t) \equiv \hat{\theta}\right|_{z=H}=\left(\frac{\mu}{H} \tanh \mu\right) \hat{\psi}^{t}(t) \quad \text { and }
$$




$$
\left.\hat{\theta}^{b}(t) \equiv \hat{\theta}\right|_{z=0}=\left(-\frac{\mu}{H} \tanh \mu\right) \hat{\psi}^{b}(t) .
$$

Expanding the potential vorticity in interior modes $q(x, y, z, t)=\hat{q}^{\mathrm{bt}}(t)+\hat{q}^{\mathrm{bc}}(t) \phi(z)$ yields

$$
\begin{aligned}
\hat{q}^{\mathrm{bt}}(t) & =-K^{2} \hat{\psi}^{\mathrm{bt}}(t), \\
\hat{q}^{\mathrm{bc}}(t) & =-K^{2} \frac{\mu^{2}+\pi^{2}}{\mu^{2}} \hat{\psi}^{\mathrm{bc}}(t) .
\end{aligned}
$$

Given the potential vorticity and surface temperature fields, (12) and (13) can be inverted to give the four streamfunction components, and the full streamfunction is thus

$$
\begin{aligned}
\psi(x, y, z, t)= & \sum_{\mathbf{K}} e^{i \mathbf{K} \cdot \mathbf{x}}\left[\hat{\psi}_{\mathbf{K}}^{\mathrm{bt}}(t)+\hat{\psi}_{\mathbf{K}}^{\mathrm{bc}}(t) \phi(z)\right. \\
& \left.+\hat{\psi}_{K}^{t}(t) \phi_{K}^{t}(z)+\hat{\psi}_{K}^{b}(t) \phi_{K}^{b}(z)\right] .
\end{aligned}
$$

The prognostic equations for the barotropic and baroclinic potential vorticity components in (13) are derived by expanding the streamfunction and potential vorticity in (1a) using (14), then projecting onto the barotropic mode by integrating in the vertical, and onto the baroclinic mode by integrating $\phi$ times the expression. Each surface temperature equation is obtained by evaluating the full advecting streamfunction in (14) at the vertical level of the surface of interest.

\section{d. Mean field projections}

The last step is to project the mean velocity onto the truncated vertical representation. The mean zonal velocity must in general satisfy (3) (but we set $V=0$ ), and to be consistent with the dynamic variables $q$ and $\theta$ we decompose the mean zonal velocity into interior and surface components $U(z)=U^{I}(z)+U^{S}(z)$. The relationships among the mean fields, however, are somewhat different than that between the eddy fields, due to the fact $U$ is independent of $x$ and $y$ (i.e., mean relative vorticity is neglected, consistent with the assumption of local homogeneity). Assumption (iv) gives us that $U^{I}=$ $U^{\mathrm{bc}} \phi(z)$, where $\phi(z)$ is given by (9). Derivatives of $U^{I}$ evaluated at 0 and $H$ vanish and therefore $U^{S}$ must satisfy the boundary conditions, given arbitrary $\Theta_{y}^{t}$ and $\Theta_{y}^{b}$. We thus demand that the surface component solves

$$
\Gamma U^{S}=A, \quad \frac{d U^{S}}{d z}(H)=-\Theta_{y}^{t}, \quad \text { and } \quad \frac{d U^{S}}{d z}(0)=-\Theta_{y}^{b} .
$$

In analogy with the decomposition of the eddy components in (4) and (6), one might expect to demand that $\Gamma U^{S}=0$. However, this can only be satisfied if $\Theta_{y}^{t}=\Theta_{y}^{b}$ (therefore it would also be impossible to separate $U^{S}$ into $U^{t}+U^{b}$ ). Instead, here we demand that the righthand side, $A$, be a constant, and that the vertical mean of the surface velocity vanish, $\left\langle U^{S}\right\rangle=0$. The result is that

$$
A=\left(\frac{f^{2}}{H N^{2}}\right) \Delta \Theta_{y}, \quad \Delta \Theta_{y} \equiv \Theta_{y}^{t}-\Theta_{y}^{b},
$$

which vanishes only when upper and lower temperature gradients are equal, and

$$
U^{S}(z)=-\Theta_{y}^{b}\left(z-\frac{H}{2}\right)+\Delta \Theta_{y}\left(\frac{z^{2}}{2 H}-\frac{H}{6}\right) .
$$

The full mean velocity is therefore $U(z)=U^{\mathrm{bc}} \phi(z)+$ $U^{S}(z)$. [A Green's function approach to the mean velocity problem is illustrated in appendix A.]

That $A \neq 0$ means that the surface velocity field $U^{S}$ induces an interior mean PV gradient; the total mean potential vorticity gradient is therefore

$$
Q_{y}=\beta-\Gamma U=\beta+L_{D}^{-2}\left[H \Delta \Theta_{y}+\sqrt{2} \pi^{2} U^{\mathrm{bc}} \cos \left(\frac{\pi z}{H}\right)\right],
$$

where $L_{D}=N H / f$ is the deformation scale and (9) was used [note that the first baroclinic deformation wavenumber, defined in (9), is $\left.\lambda=\pi / L_{D}\right]$.

\section{e. The two-mode, two-surface model}

Putting all the prior results together, the full set of spectral prognostic equations can now be written as

$$
\begin{aligned}
& \hat{\theta}_{t}^{t}+\hat{J}\left(\left.\hat{\psi}\right|_{z=H}, \hat{\theta}^{t}\right)+i k\left[U(H) \hat{\theta}^{t}+\left.\Theta_{y}^{t} \hat{\psi}\right|_{z=H}\right]=0, \quad \text { (18a) } \\
& \hat{\theta}_{t}^{b}+\hat{J}\left(\left.\hat{\psi}\right|_{z=0}, \hat{\theta}^{b}\right)+i k\left[U(0) \hat{\theta}^{b}+\left.\Theta_{y}^{b} \hat{\psi}\right|_{z=0}\right]=\left.r K^{2} \hat{\psi}\right|_{z=0},
\end{aligned}
$$

$$
\begin{aligned}
& \hat{q}_{t}^{\mathrm{bt}}+\hat{J}\left(\langle\hat{\psi}\rangle, \hat{q}^{\mathrm{bt}}\right)+\hat{J}\left(\langle\phi \hat{\psi}\rangle, \hat{q}^{\mathrm{bc}}\right) \\
& +i k\left[\langle\phi U\rangle \hat{q}^{\mathrm{bc}}+\left(\beta-\Gamma U^{S}\right)\langle\hat{\psi}\rangle+\lambda^{2} U^{\mathrm{bc}}\langle\phi \hat{\psi}\rangle\right]=0 \\
& \hat{q}_{t}^{\mathrm{bc}}+\hat{J}\left(\langle\phi \hat{\psi}\rangle, \hat{q}^{\mathrm{bt}}\right)+\hat{J}\left(\langle\phi \phi \hat{\psi}\rangle, \hat{q}^{\mathrm{bc}}\right) \\
& \quad+i k\left[\langle\phi U\rangle \hat{q}^{\mathrm{bt}}+\langle\phi \phi U\rangle \hat{q}^{\mathrm{bc}}+\left(\beta-\Gamma U^{S}\right)\langle\phi \hat{\psi}\rangle\right. \\
& \left.\quad+\lambda^{2} U^{\mathrm{bc}}\langle\phi \phi \hat{\psi}\rangle\right]=0
\end{aligned}
$$

where $\hat{J}$ is shorthand for the sum over wavenumbers of the Jacobian terms, and the streamfunction evaluated at the upper and lower surfaces, respectively, is 


$$
\begin{gathered}
\left.\hat{\psi}\right|_{z=H}=\hat{\psi}^{\mathrm{bt}}-\sqrt{2} \hat{\psi}^{\mathrm{bc}}+\hat{\psi}^{t}+\hat{\psi}^{b} \operatorname{sech} \mu \\
\left.\hat{\psi}\right|_{z=0}=\hat{\psi}^{\mathrm{bt}}+\sqrt{2} \hat{\psi}^{\mathrm{bc}}+\hat{\psi}^{t} \operatorname{sech} \mu+\hat{\psi}^{b} .
\end{gathered}
$$

The vertical integrals and projections of the total streamfunction and mean shear onto the baroclinic mode (e.g., $\langle\phi \hat{\psi}\rangle)$ are derived and stated in appendix B.

Equations (18) sacrifice the ability to represent high vertical modes of the interior flow, but retain an accurate description of surface motions, even when such motions have very small vertical penetration into the interior. The projection onto a truncated set of interior modes, plus surface modes, allows for a compact model that can be numerically integrated with much greater efficiency than a high-vertical resolution gridded model. The goal in developing this model is twofold: (i) to derive a simple model that retains all basic types of baroclinic instability, and (ii) to demonstrate that augmentation of the two-layer model with surface modes is sufficient to explain the spectrum of energy in the atmospheric mesoscales. The model is not sufficient to explore more complex vertical structures, and this will be taken up in a future paper.

\section{Linear instability calculation}

Assuming horizontally constant, baroclinic zonal mean flow, the quasigeostrophic equations in (1) are linearly unstable to small perturbations under at least one of the following conditions (Charney and Stern 1962; Pedlosky 1964): (i) $Q_{y}=0$, and $\Theta_{y}^{t}$ and $\Theta_{y}^{b}$ are both nonzero and have the same sign (as in the model of Eady 1949); (ii) $Q_{y}$ changes sign in the interior and the boundary gradients are zero (as in the model of Phillips 1954); or (iii) $Q_{y} \neq 0$ and either the upper surface gradient has the same sign, or the lower surface gradient has the opposite sign as $Q_{y}$ (as in the model of Charney 1947). The standard two-layer model of Phillips admits only instabilities of the second type, yet has arguably been more widely used than either the Charney or Eady model, due to its analytical tractability, wide parameter range possibilities, inclusion of $\beta$, and the ease with which it can be numerically simulated. The TMTS model in (18) derived in section 2 is intended to retain those positive features of the two-layer model while additionally admitting the two missing instability types [(i) and (iii)]. Here we have the following goals: to demonstrate that all of types (i)-(iii) are captured in the two-mode, two-surface model presented in section 2 (and this requires redoing some standard calculations); and to compute the linear instability of the flow that will be used to drive the nonlinear turbulence simulations presented in section 4 .
To compute the baroclinic instability of the TMTS model, the nonlinear terms are neglected and a normalmode wave solution is assumed: $\left(\hat{\psi}^{t}, \hat{\psi}^{b}, \hat{\psi}^{\mathrm{bt}}, \hat{\psi}^{\mathrm{bc}}\right)=$ $\Re \varphi \exp (-i \omega t)$, where $\varphi=\left(\hat{\varphi}^{t}, \hat{\varphi}^{b}, \hat{\varphi}^{\mathrm{bt}}, \hat{\varphi}^{\mathrm{bc}}\right)$ and the meridional wavenumber $\ell$ is set to 0 . Specifically, we solve the eigenvalue problem $c \varphi=\mathbf{A} \varphi$ where $\mathbf{A}$ is a $4 \times 4$ matrix (given in appendix C) and $c=\omega / k$. The growth rate $\omega_{i}=$ $k c_{i}$ of unstable modes depends on $\beta$, the magnitude of the internal velocity shear $U^{\mathrm{bc}}$, and on the boundary temperature gradients $\Theta_{y}^{t, b}$. We nondimensionalize the parameter space of the problem with horizontal length scale $L_{D}$, vertical length scale $H$, and a velocity scale $U_{0}$. Horizontal wavenumbers $K$ are already expressed nondimensionally as $\mu=K L_{d}$ almost everywhere they appear. The nondimensional Coriolis gradient is $\tilde{\beta} \equiv$ $\beta L_{d}^{2} / U_{0}$ and the velocity parameters of the problem are $U^{\mathrm{bc}} / U_{0}$ and $\left(H / U_{0}\right) \Theta_{y}^{t, b}=-\left(H / U_{0}\right) U_{z}^{t, b}$.

Figures 2 and 3 show numerically computed growth rates and amplitudes of eigenfunctions of the linearized TMTS model as functions of $\mu$ and $\tilde{\beta}$. Figure 2a shows the growth rates given equal, nonzero boundary temperature gradients $\left(H / U_{0}\right) \Theta_{y}^{t}=\left(H / U_{0}\right) \Theta_{y}^{b}=-1$, and zero interior shear, $U^{\mathrm{bc}}=0$. The Eady problem corresponds to the line $\tilde{\beta}=0$, and along this line, $\max \left(\omega_{i}\right) L_{d}$ l $U_{0} \approx 0.31$, as expected. For $\tilde{\beta} \neq 0$ boundary gradients interact with $Q_{y}=\beta$ in the interior, which results in Charney-type instabilities at small scales and a truncated Green (1960) mode at large scales (see also Lindzen 1994, who considered the effects of altering the mean state to retain 0 interior PV with $\beta$ ). Figure $2 \mathrm{~b}$ shows the amplitudes, as functions of height, that correspond to the fastest-growing modes at various locations in the $(\mu, \tilde{\beta})$ plane, as indicated by symbols in Fig. 2a. The plus symbol, for example, corresponds to the location of maximum growth in the Eady problem $(\tilde{\beta}=0)$, and has the expected symmetric amplitude, peaked at the boundaries [cosh $\mu z / H-\left(U_{0} / \mu c\right) \sinh$ $\mu z / H]$. The triangle is located in the Green-mode region, and has a vertical structure that is comparable to Fig. 6 of Green (1960). The circle is in the bottom Charney-mode region and its vertical structure is, likewise, comparable to that expected for the Charney problem (see, e.g., Pedlosky 1987, his Fig. 7.8.5).

Figure $2 \mathrm{c}$ shows the growth rates for the pure interior shear problem, with $\Theta_{y}^{t}=\Theta_{y}^{b}=0$ and $U^{\mathrm{bc}} / U_{0}=$ $-1(\sqrt{2} \pi)$ and Fig. $2 \mathrm{~d}$ shows its vertical structure. This is the standard two-mode Phillips problem with max $\left(\omega_{i}\right) L_{d} /\left|U^{\mathrm{bc}}\right|=(\sqrt{2}-1) \pi$, and so the chosen value of $U^{\mathrm{bc}} / U_{0}$ gives $\max \left(\omega_{i}\right) L_{d} / U_{0}=1-1 \sqrt{2}=0.29$, which is close to the maximum growth rate for the Eady problem. As expected, there is no longwave cutoff for $\tilde{\beta}=0$. For large enough $\tilde{\beta}$, instability is suppressed, and the amplitude is peaked at the boundaries, but is large 


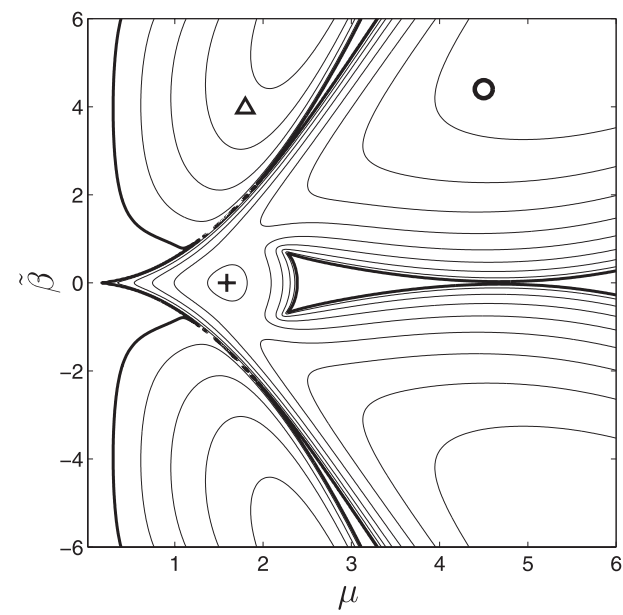

(a)
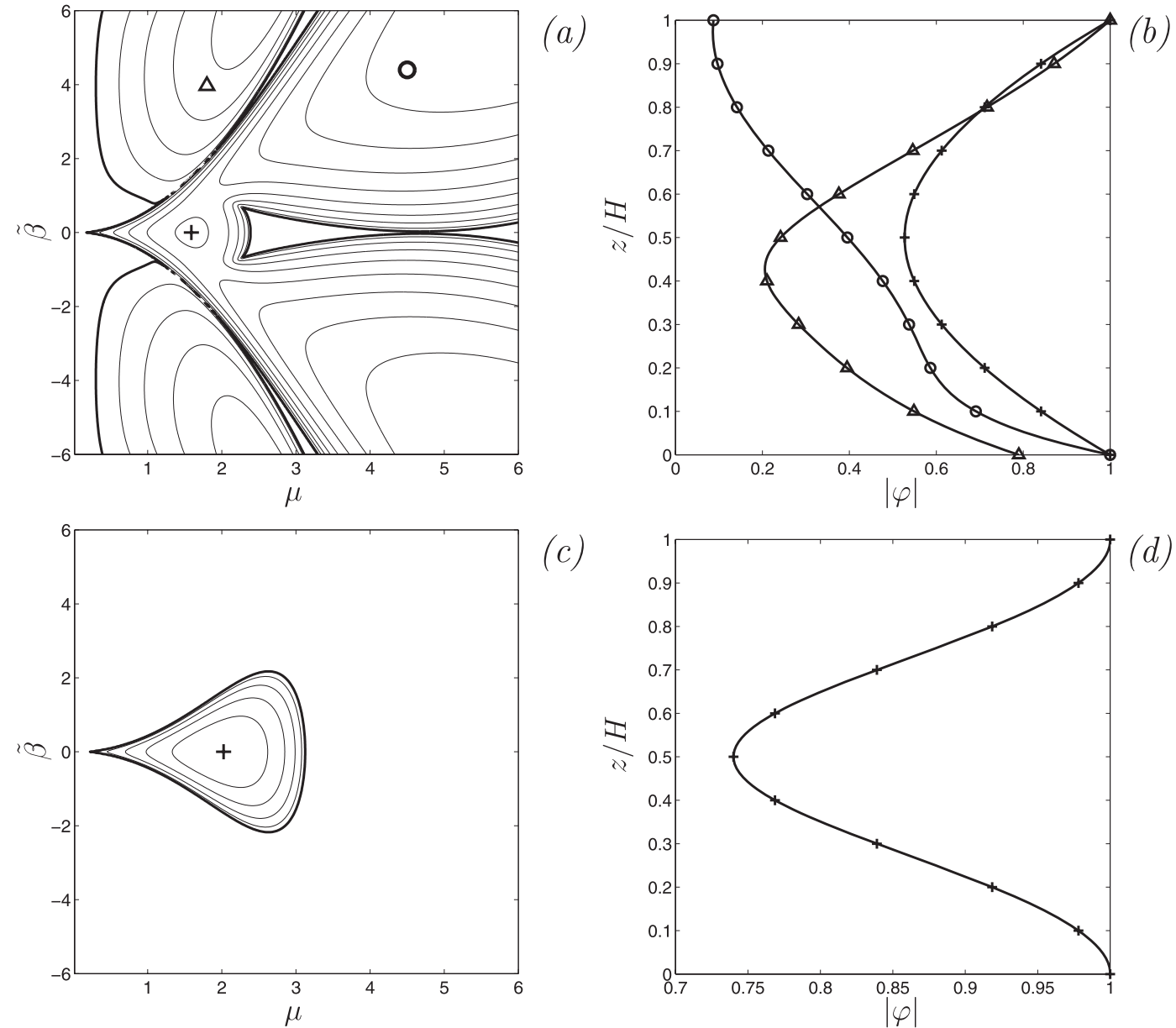

(c)

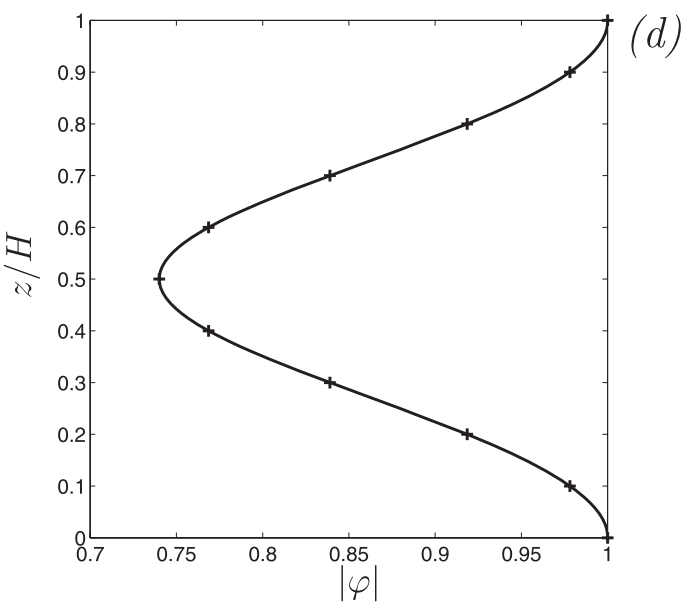

FIG. 2. Growth rates vs nondimensional $\beta$ and zonal wavenumber are plotted for (a) an Eady-like instability (when $\tilde{\beta}=0$ ) with only mean surface gradients and (c) a Phillips-type instability with only mean interior gradients. Contour values are vary linearly from 0.05 (thick line) to 0.4 at 0.05 intervals. Note that growth rates have been nondimensionalized by $U_{0} / L_{d}$. (b), (d) The amplitudes as a function of $z$ for the instability points with the same symbols as in (a) and (c), respectively.

throughout the depth of the fluid. Figure 3a shows the growth rate for equally weighted surface and interior forcing $\left(H / U_{0}\right) \Theta_{y}^{t}=\left(H / U_{0}\right) \Theta_{y}^{b}=-1$ and $U^{\mathrm{bc}} / U_{0}=$ $-1 /(\sqrt{2} \pi)$; comparison to Fig. 2 a shows that the effect of the interior shear in this case is primarily to suppress growth at small scales, for small values of $\tilde{\beta}$. The vertical structure of the amplitudes for the three apparent peaks are similar to those in Figs. 2a,b, except that the amplitude corresponding to the $\tilde{\beta}=0$ instability is larger. Figure $3 \mathrm{c}$ shows the growth rate for a mean state with an upper-surface temperature gradient $\left(H / U_{0}\right) \Theta_{y}^{t}=-1 / 2$, a vanishing lower-surface temperature gradient, and an interior shear $U^{\mathrm{bc}} / U_{0}=-4 /(\sqrt{2} \pi), 4$ times larger than the interior shear used in Fig. 3a. This mean state is used in the central nonlinear simulation discussed in the next section. Removing the bottom temperature gradient has suppressed the large-scale Green modes, and left only type (iii) (Charney) instabilities at small scales. The asymmetry at small scales occurs because the upper-surface temperature gradient and the interior PV gradient must be of the same sign [consider the PV gradient in Eq. (17)].

\section{Nonlinear simulations}

Here we report on the results of a series of simulations made with the fully nonlinear TMTS model in (18), using parameters relevant to the midlatitude atmosphere. In all cases, we set the dimensional parameters $U_{0}=H=1$, and $L=2 \pi$, so that wavenumber 1 fills the domain. In the primary series, the interior shear and bottom temperature gradient are held constant at $U^{\mathrm{bc}}=-4 /(\sqrt{2} \pi)$ and $\Theta_{y}^{b}=0$, respectively, but 


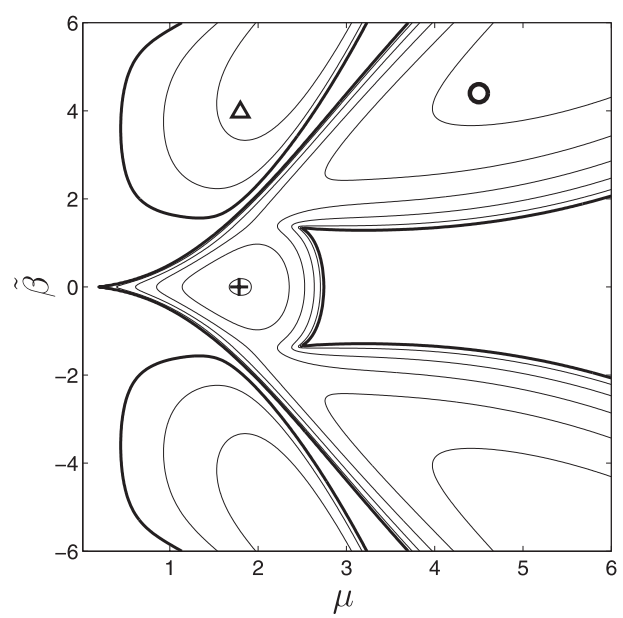

(a)
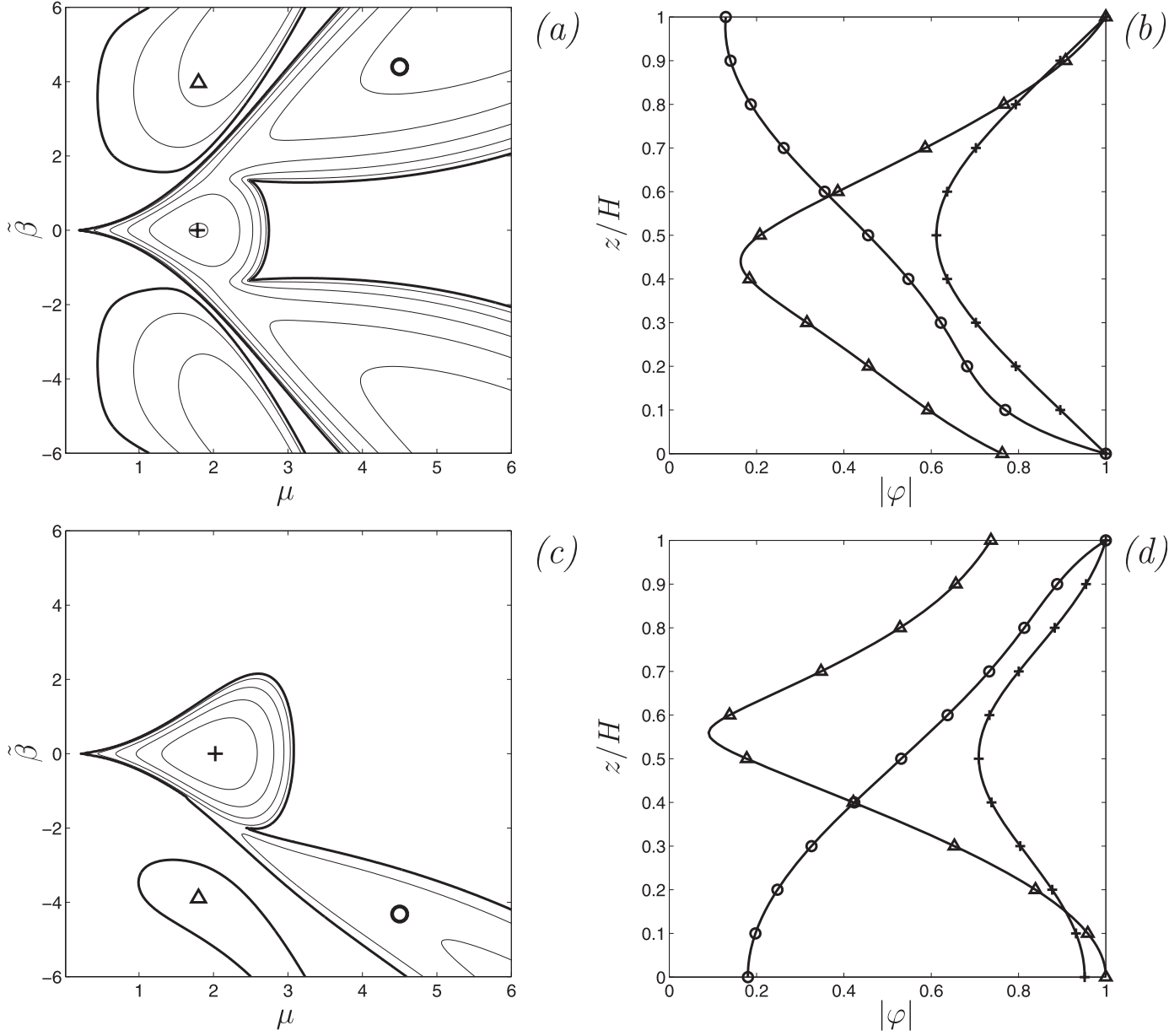

(c)

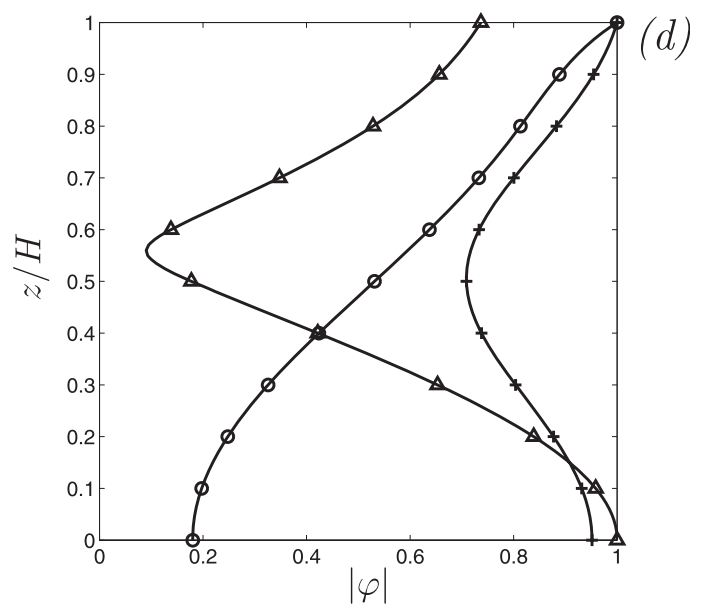

FIG. 3. Growth rates and eigenfunctions as in Fig. 2, but for mixed surface and interior mean gradients. (a), (b) Equal surface and interior mean gradients. (c), (d) Linear instabilities for the parameters used in the nonlinear simulations in section 4 .

the upper-surface temperature gradient is varied from $\Theta_{y}^{t}=-1 / 4$ to -2 . The model is pseudospectral, fully dealiased, and is run at a maximum resolution equivalent to $2048^{2}$ grid points $\left(K_{\max }=1027\right)$. The model deformation wavenumber $K_{D}=1 / L_{D}=2$, to allow a wide forward cascade. The Coriolis gradient is set to $\tilde{\beta}=\beta L_{D}^{2} / U_{0}=1$ and the Ekman drag is $r / U_{0} \approx 0.18$ (the drag is varied slightly with $\Theta_{y}^{t}$ in to keep the energy injection rate nearly constant among the runs). The baroclinic growth rates for the mean states in this series of simulations are all nearly the same as shown in Fig. 3c (which corresponds to the case with $\Theta_{y}^{t}=-1 / 2$ ). A highly scale-selective exponential cutoff filter absorbs the forward cascade (it is explicitly restricted to act only on wavenumbers $K>2 K_{\max } / 3$, but in fact only affects a much smaller range of wavenumbers close to $K_{\max }$ ). The details of this filter are discussed in Smith et al. (2002).

Figure 4 shows snapshots of the PV and temperature at the upper surface for the simulation with $\Theta_{y}^{t}=-1 / 2$.
Figure 4a shows the entire PV field, Fig. 4b shows the entire temperature field, while Figs. $4 c$,d show close-ups of the fields inside the dashed boxes of the top panels. At large scales the PV and temperature are anticorrelated and dominated by the PV, while at small scales the surface dynamics are revealed: small-scale vortices (due to secondary roll-up of filaments; Held et al. 1995) are widespread in the temperature field, and appear to actively stir the PV. The anticorrelation between PV and temperature is consistent with Lapeyre and Klein (2006), who show that if the forcing of interior PV and surface temperature are the same, then the anomalies will be strongly correlated or anticorrelated, depending on $Q_{y}$ and $\Theta_{y}$, even if they are not advected by exactly the same flow.

Figure 5 shows the total kinetic energy spectrum (for the same run with $\Theta_{y}^{t}=-1 / 2$ ), after equilibration and averaged over many eddy turnover times, at the top surface $E(K, z=H)=K^{2}|\hat{\psi}(H)|^{2}$ as well as the 

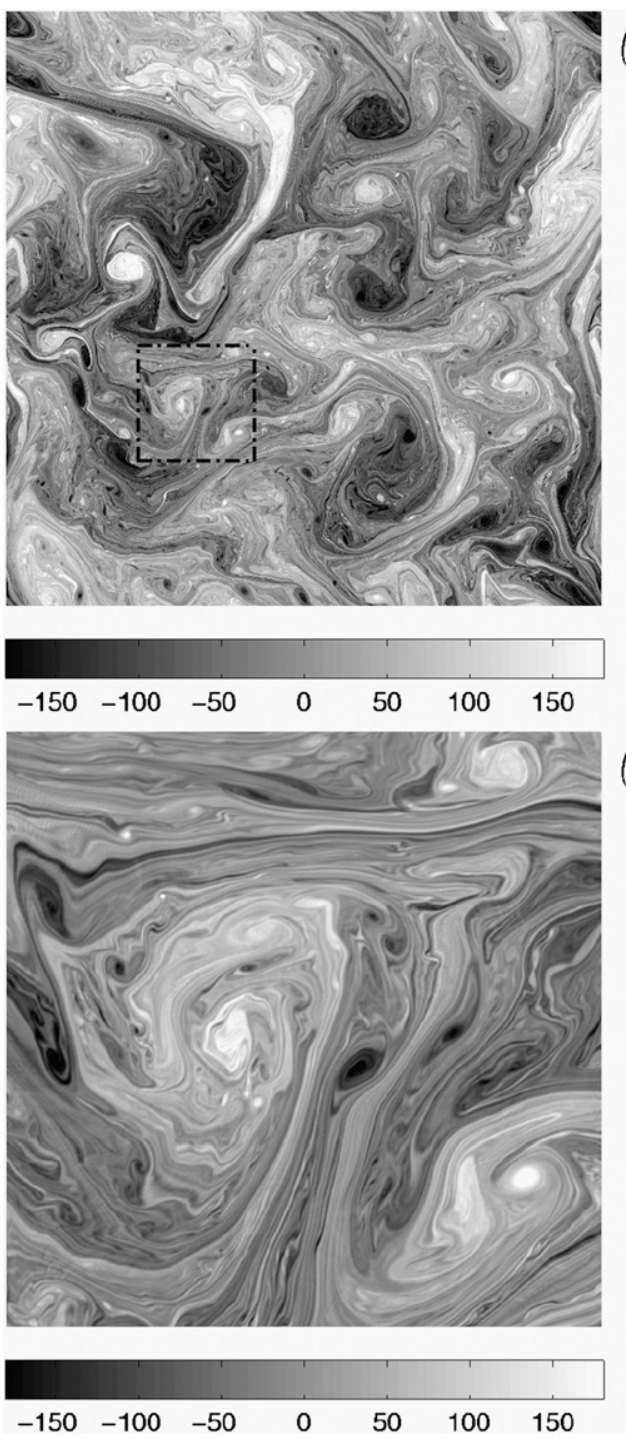

(a)

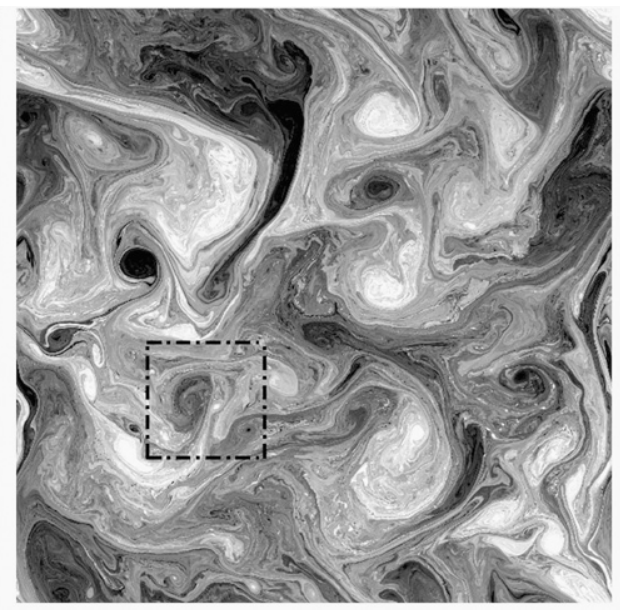

(b)

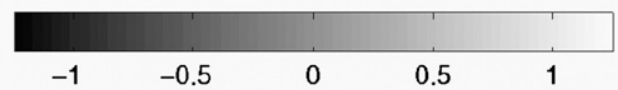

(c)

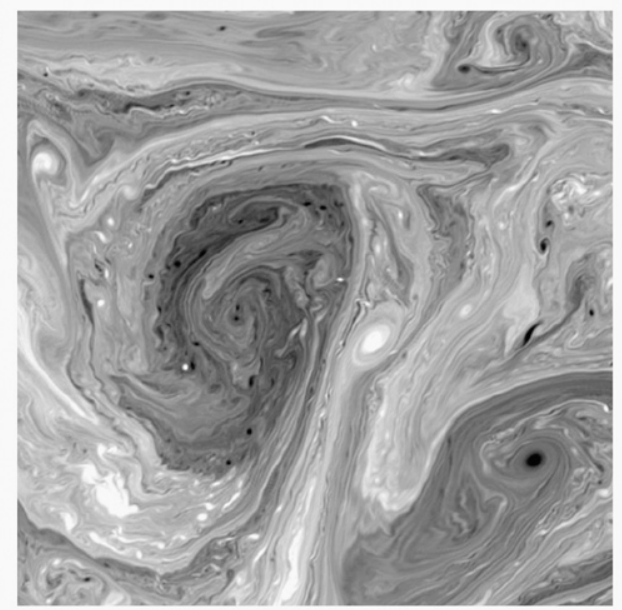

(d)

FIG. 4. Snapshots of (a) [close-up in (c)] PV and (b) [close-up in (d)] temperature at the top surface for the $\Theta_{y}^{t}=-0.5$ case. At large scales $q(H)$ and $\theta^{t}$ are anticorrelated and driven by the PV dynamics. At small scales $q(H)$ is dominated by the dynamics of vortices present in $\theta^{t}$.

components that contribute to the total kinetic energy, plotted against horizontal wavenumber. The dash-dot line is the barotropic kinetic energy $K^{2}\left|\hat{\psi}^{\mathrm{bt}}\right|^{2}$, which is driven primarily by the interior shear $U^{\text {bc }}$ and has a steep $K^{-3}$ slope as a result of enstrophy cascading to small scales. The dashed line is the spectrum of APE at the upper surface, which is equal to the kinetic energy of the surface streamfunction $K^{2}\left|\hat{\psi}^{t}\right|^{2}$ and cascades forward with a shallow $K^{-5 / 3}$ slope [also see Gkioulekas and Tung (2007a) for a derivation of equipartition between KE and APE in SQG turbulence and Gkioulekas and Tung (2007b) for a proof of the cascade direction]. The solid line is the total kinetic energy spectrum at the upper surface, which is apparently a superposition of the barotropic and surface-induced spectra (with some influence from the baroclinic kinetic energy at large scales), perhaps as expected from the PV and temperature fields in Fig. 4. There is a transition from $K^{-3}$ interior-dominated dynamics to $K^{-5 / 3}$ surface-dominated dynamics at a wavenumber that depends on the relative energy levels in the surface and interior modes, which in turn depend on the relative strengths of the surface and interior baroclinic forcings. We also note that, because the interior dynamics in the numerical model are truncated at the first baroclinic mode, the interior APE is concentrated at $z=H / 2$, and so the simulated APE lacks a $K^{-3}$ slope at large scales at or near the upper surface. 


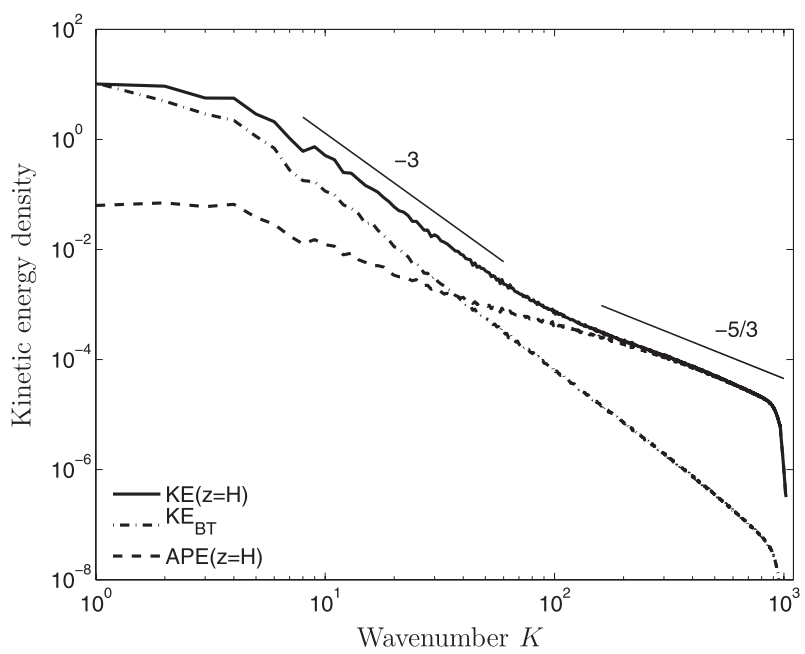

FIG. 5. Energy densities as a function of horizontal wavenumber for the $\Theta_{y}^{t}=-0.5$ simulation. The kinetic energy density at the top surface (thick solid) exhibits a transition from -3 where barotropic kinetic energy (dash-dot) dominates to $-5 / 3$ at $k \approx 100$ as the variance of temperature (long dashed) begins to dominate the forward cascade.

\section{a. The transition scale}

Figure 6 shows the upper-surface kinetic energy spectra for each of the series of simulations in which $\Theta_{y}^{t}$ is varied from $-1 / 4$ to -2 . It is apparent that the transition scale between the steep large-scale spectrum and the shallow small-scale spectrum is controlled by $\Theta_{y}^{t}$. The particular dependence of the transition scale on the parameters of the problem can be understood as follows. The upper-level energy spectrum in the forward enstrophy cascade has the form

$$
\mathcal{E}(K)=\mathcal{C}_{E} \eta^{2 / 3} K^{-3},
$$

where the rate of enstrophy transfer at $z=H$ is

$$
\eta=-Q_{y}(H) \overline{v q}_{z=H} \equiv \kappa_{q} Q_{y}(H)^{2} .
$$

The overbar denotes a horizontal average, $C_{E}$ is a Kolmogorov constant, and we have defined a PV diffusivity $\kappa_{q}$. The cascade of temperature variance at the upper surface leads to an available potential energy spectrum of the form

$$
\mathcal{A}(K)=\mathcal{C}_{A} \epsilon^{2 / 3} K^{-5 / 3},
$$

where the relevant energy flux is

$$
\epsilon=-\left.\frac{f^{2} \Theta_{y}^{t}}{N^{2}} \overline{v \theta^{t}}\right|_{z=H} \equiv \kappa_{\theta}\left(\frac{f \Theta_{y}^{t}}{N}\right)^{2} .
$$

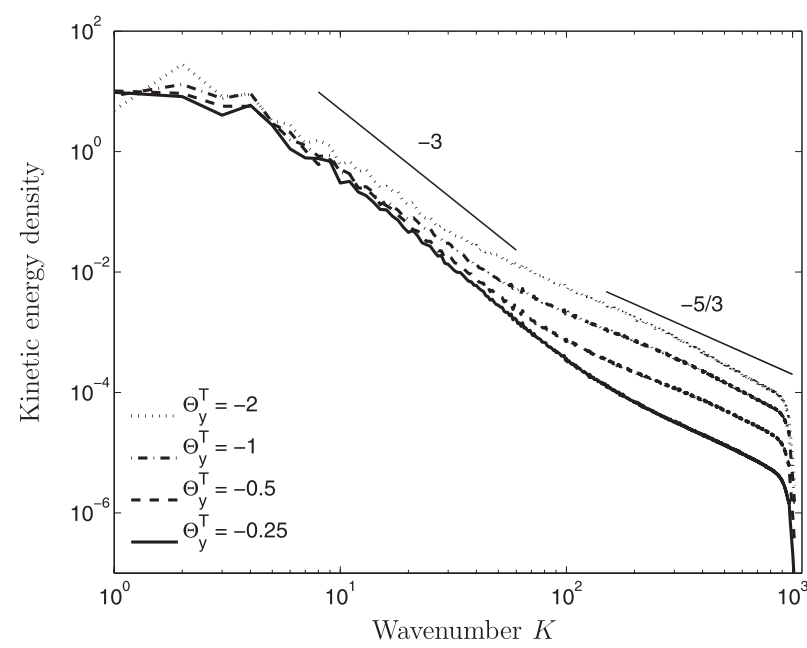

FIG. 6. Kinetic energy spectra at $z=H$ with $\Theta_{y}^{t}=-2,-1,-0.5$, and $-0.25, U^{\mathrm{bc}}=-4 /(\pi \sqrt{2})$ and $H=1$ at $2048^{2}$ resolution. Thin lines are $k^{-5 / 3}$ and $k^{-3}$ for reference. The small-scale spectra are approximately $11 k^{-5 / 3}, 5 k^{-5 / 3}, 1.5 k^{-5 / 3}$, and $0.45 k^{-5 / 3}$.

Here we have defined a second diffusivity $\kappa_{\theta}$ for the temperature, and a second Kolmogorov constant for the temperature cascade.

Assuming equal diffusivities $\kappa_{q} \simeq \kappa_{\theta}$ and Kolmogorov constants $\mathrm{C}_{E} \simeq \mathrm{C}_{A}$, and solving for the wavenumber where the two cascades are equal, one finds the upperlevel transition wavenumber

$$
K_{\text {trans }} \simeq \frac{N}{f}\left|\frac{Q_{y}(H)}{\Theta_{y}^{t}}\right| .
$$

It is instructive to rewrite this expression as

$$
K_{\text {trans }} \simeq\left|L_{C}^{-1}+L_{D}^{-1} \frac{U_{z}(0)-U_{z}(H)-\sqrt{2} \pi^{2} U^{b c} / H}{\left|U_{z}(H)\right|}\right|,
$$

where (3) and (17) were used to replace the PV and temperature gradients with shears and

$$
L_{C}=\frac{f}{N} \frac{\left|U_{z}(H)\right|}{\beta}
$$

is the Charney length (see, e.g., Pedlosky 1987). The second expression for $K_{\text {trans }}$ now has a form similar to that of the transition wavenumber found by Tulloch and Smith (2006), $L_{D}^{-1}=f / N H$, except that here (pulling out a factor $f / N)$ there are two vertical scales, added in reciprocal: the Charney depth $\left(h_{C}=f L_{C} / N\right)$ and a 


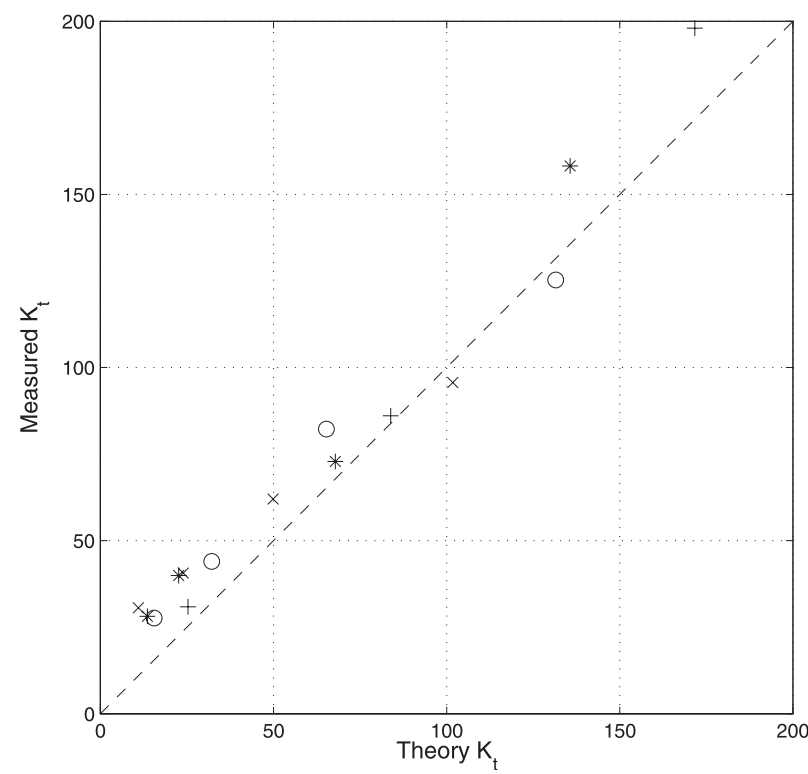

FIG. 7. The measured transition wavenumber for all simulations, defined as where the slope is $k^{-7 / 3}$, compared with the prediction from (20). We set $L=2 \pi, U_{0}=H=1$ for all runs. Asterisks: $\Theta_{y}^{t}=\Theta_{y}^{b}=-5,-3,-1,-0.5, U^{\mathrm{bc}}=-1, \tilde{\beta}=3, K_{\mathrm{D}}=4$; plus signs: same as asterisks, but $\Theta_{y}^{t}=-5$ for each; circles: $\Theta_{y}^{t}=-2$, $-1,-0.5,-0.25, \Theta_{y}^{b}=0, U^{\mathrm{bc}}=-4 /(\sqrt{2} \pi), \tilde{\beta}=1, K_{D}=2$; crosses: $\Theta_{y}^{t}=-2,-1,-0.5,-0.25, \Theta_{y}^{b}=0, U^{\mathrm{bc}}=-0.7, \tilde{\beta}=3$, and $K_{D}=2$.

second term corresponding to the fluid depth $H$ times the relative ratio of surface to total shears. In the limit of no interior or bottom shear, and assuming $h_{C} \ll H$, the vertical scale is just the Charney depth, and $K_{\text {trans }} \simeq$ $L_{C}^{-1}$. In the limit of $\beta=0$, the transition scales with the inverse deformation scale, and if additionally $U_{z}(H) \gg$ $U^{\mathrm{bc}} / H$, then the vertical scale is $H\left(K_{\text {trans }} \simeq L_{D}^{-1}\right)$, as found in the simpler model of Tulloch and Smith (2006).

The scaling prediction in (20) is tested against the "measured" transition wavenumbers for all simulations performed (including a third series identical to the second series, but where the bottom temperature gradient is held fixed at $\Theta_{y}^{b}=5$ ) in Fig. 7 (see caption for details of the transition-scale computation). The theory apparently captures the variation of transition scale with surface shear quite well. There is a bias toward underpredicting the measured transition wavenumber when $K_{\text {trans }}$ is small, which is perhaps due to halting scale (or drag) effects in the numerical model, which are not accounted for in the theory. We also check here that the results are independent of horizontal resolution. Figure 8 shows the resulting surface energy spectra for a series of simulations in which all parameters are held constant $\left(\Theta_{y}^{t}=-0.5\right)$, but horizontal resolution is successively reduced. The results confirm that the transi- tion from -3 to $-5 / 3$ is independent of numerical resolution, as well as small-scale filtering.

We can check that the surface energy exhibits an inertial range cascade by computing the energy flux directly, as a function of the wavenumber. For the series of simulations in which the upper-surface temperature gradient is varied, the surface fluxes of available potential energy,

$$
\epsilon(K)=\frac{f^{2}}{N^{2}} \int_{0}^{K} \hat{\theta}^{t} \hat{J}\left(\hat{\psi}, \hat{\theta}^{t}\right) d K^{\prime}
$$

are shown in Fig. 9. The fluxes are plainly constant, as suggested.

\section{b. Atmospheric forcing}

Using long-term monthly mean data from the NCEP reanalysis simulations, a mean wind profile is computed by averaging data from $45^{\circ} \mathrm{N}$ temporally and zonally. The meridional potential temperature gradients from the 1000- and 200-mb data (corresponding to $H \simeq 9.7$ $\mathrm{km}$ ) are computed from zonal and temporal averages at the same latitude, from which the mean upper- and lower-level shears are inferred from thermal wind balance, and the profile $U^{S}$ is then computed from the shears. The interior first baroclinic mean zonal wind is then approximately the difference between the NCEP data profile and the surface-induced zonal wind. The resulting surface shears are $U_{z}(H)=5.6 \times 10^{-4} \mathrm{~s}^{-1}$ and $U_{z}(0)=2.1 \times 10^{-3} \mathrm{~s}^{-1}$, and the interior baroclinic velocity is $U^{\mathrm{bc}}=-2.6 \mathrm{~m} \mathrm{~s}^{-1}$, corresponding to an interior shear $\sqrt{2} \pi^{2} U^{\mathrm{bc}} / H=3.7 \times 10^{-3} \mathrm{~s}^{-1}$. Using a typical stratification $N=10^{-2} \mathrm{~s}^{-1}$, one finds $L_{C}=360 \mathrm{~km}$, $L_{D}=950 \mathrm{~km}$, and so Eq. (20) gives $K_{\text {trans }} \simeq 1 / 77 \mathrm{~km}^{-1}$ (or the transition wavelength $\simeq 480 \mathrm{~km}$ ) as the transition wavenumber predicted by our scaling theory, which is quite near the observed atmospheric transition wavelength of about $450 \mathrm{~km}$.

These mean values are used in a simulation, the results of which are shown in Fig. 10. Figure 10a shows the spectra of kinetic energy at the upper surface, the available potential energy, and the barotropic kinetic energy. The structure is similar to the spectra in Fig. 5. The bottom axis is the dimensional wavelength, for comparison with the Nastrom-Gage spectrum presented in Fig. 1. The transition wavelength in the simulation is near $300 \mathrm{~km}$, somewhat smaller than that predicted above (and smaller than the observed transition wavelength), but is consistent with the bias of underpredicting the transition wavenumber when $K_{\text {trans }}$ is small, as shown in Fig. 7 and discussed above. Note 


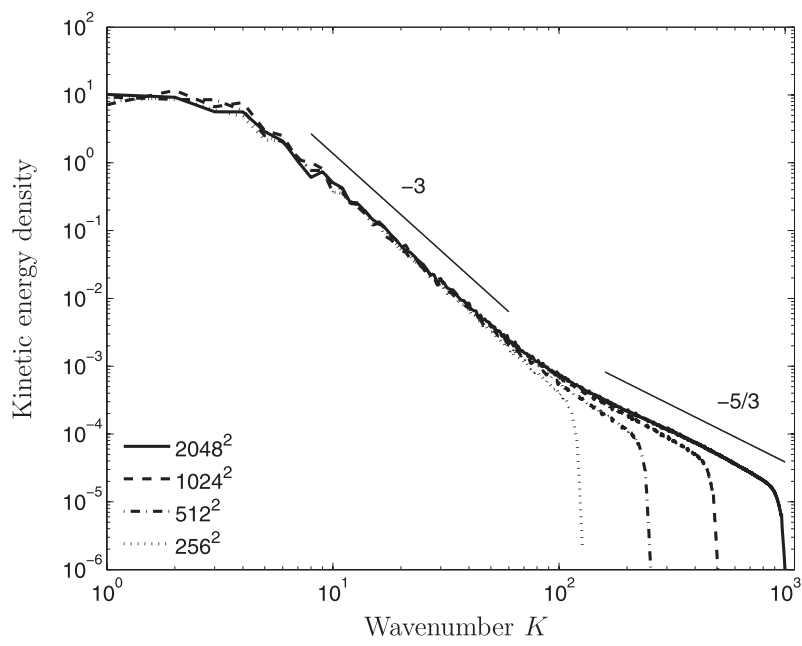

FIG. 8. Kinetic energy spectra at $z=H$ with $\Theta_{y}^{t}=-0.5$ and $K_{D}=2$, computed at different horizontal resolutions.

that we have made coarse approximations in choosing our atmospheric parameters by averaging zonally at a particular latitude and pressure level, so it is not surprising that there is a discrepancy. The overall energy level of our simulation is higher than the observed level, and the temperature variance is less. However, it should be restated that this is an idealized, doubly periodic model, designed to represent one aspect of the turbulent structure of the synoptic- and mesoscales. The large-scale forcing and dissipation are crudely represented, and the interior flow is truncated to include only two vertical modes.

Cho and Lindborg (2001) found the spectral energy flux in the MOZAIC data to be $\epsilon=6 \times 10^{-5} \mathrm{~m}^{2} \mathrm{~s}^{-3}$ just above the tropopause, while Dewan (1997) notes that observed stratospheric energy fluxes range from $1 \times 10^{-6}$ to greater than $1 \times 10^{-4} \mathrm{~m}^{2} \mathrm{~s}^{-3}$. For comparison, we compute the flux from this atmosphericparameter run and find the spectral flux of available potential energy at the surface to be $\epsilon=8 \times 10^{-5} \mathrm{~m}^{2} \mathrm{~s}^{-3}$, which is within the observed range.

Last, note that the surface energy is expected to decay away from the surface, over a depth scale proportional to $K N / f$, for $K>K_{\text {trans. }}$ Below this scale depth, the interior spectrum should be dominated by the -3 slope interior dynamics. Figure 10b shows plots of the spectra at various heights at and below the upper surface. The structure is remarkably similar to that found by Hamilton et al. (2008) (see also Takahashi et al. 2006) in very high-resolution global circulation simulations, however it stands in contrast with the simulations of Skamarock (2004) and Skamarock and Klemp (2008). The source of the discrepancies between those sets of simulations is not clear at present.

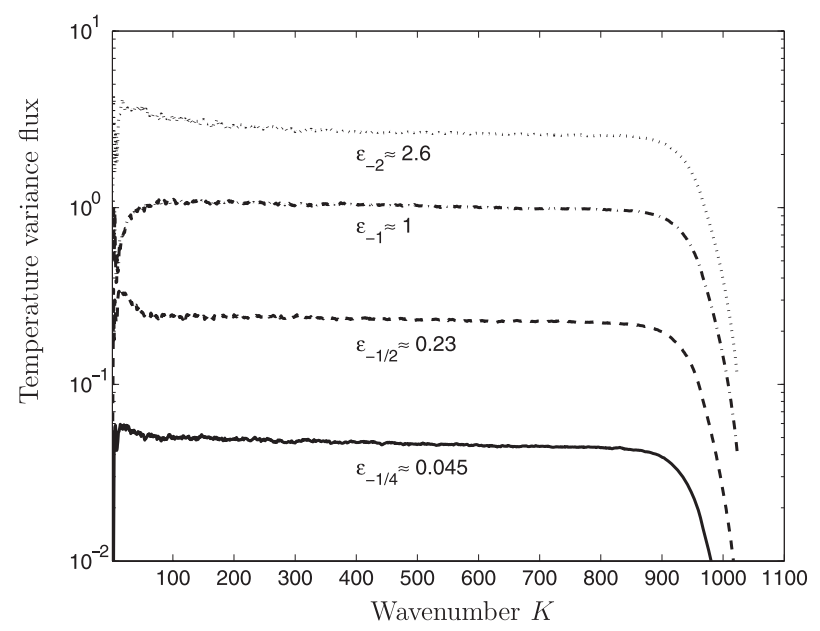

FIG. 9. Measured temperature variance fluxes for $\Theta_{y}^{t}=-2$, $-1,-0.5$, and -0.25 are $\epsilon \approx 2.6,1,0.23$, and 0.045 , respectively. Approximate values of Kolmogorov's constant for these transfer fluxes are $C_{T} \approx 5.8,5,4$, and 3.6, respectively, which are obtained from measuring the magnitude of the $k^{-5 / 3}$ part of the spectra in Fig. 6.

\section{Discussion}

We have demonstrated that a balanced model that properly represents surface buoyancy dynamics will produce a robust forward cascade along its boundaries, with a spectrum that exhibits a shallowing from -3 to $-5 / 3$ slope, consistent with the observed atmospheric kinetic energy spectrum. The TMTS model consists of four streamfunction modes: the barotropic and baroclinic interior modes due to potential vorticity in the interior and top and bottom surface modes due to potential temperature on the boundaries. The full streamfunction is a superposition of these modes because the associated inversion problem is linear. Depending on what baroclinic forcing is applied all three of the classical baroclinic instability types (i.e., Charney, Eady, and Phillips) can be excited. The transition scale in this model is set by the ratio between the horizontal temperature gradients at the upper and lower boundaries and the internal shear, since these are the drivers of energy generation for the boundary and interior spectral cascades. Using midlatitude atmospheric parameters and mean gradients (at least as well as such can be represented in this truncated model) produces a transition scale near the observed scale.

The forward energy cascade near the vertical boundaries has implications in both the atmosphere and ocean. In the atmosphere, as we have shown here, the surface modes may be responsible for the transition from steep to shallow slope in the kinetic energy cascade. In the ocean where stratification and shear are surface 

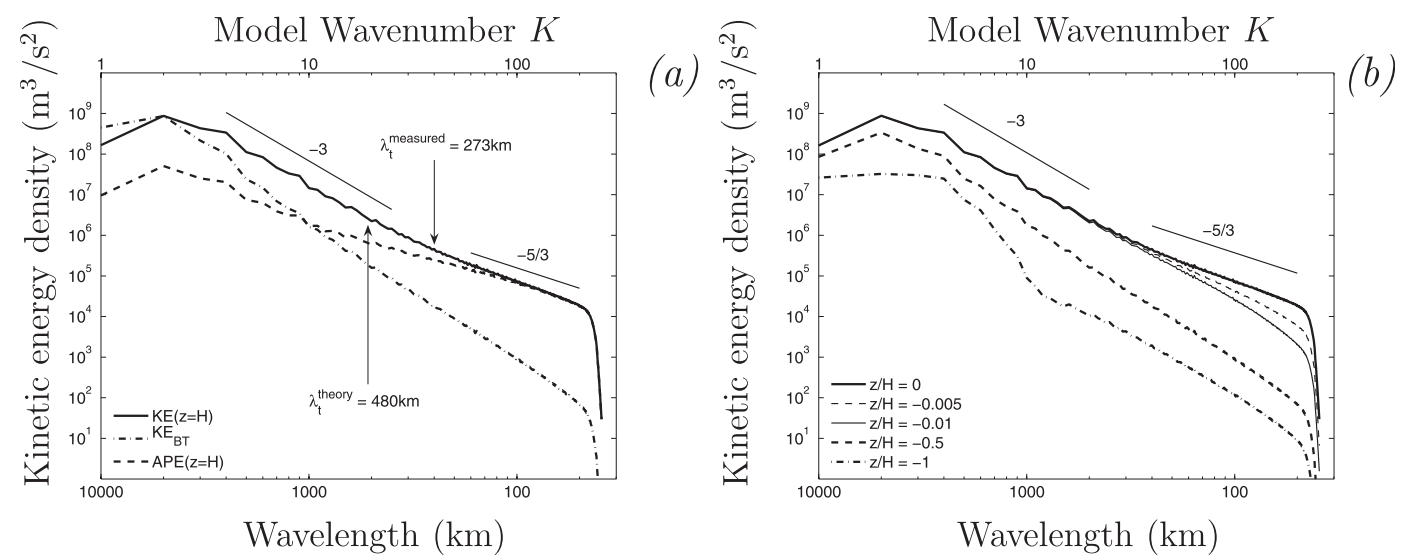

FIG. 10. (a) The spectra using zonally and temporally averaged winds from NCEP at $45^{\circ} \mathrm{N}$. Shown are the kinetic energy at the top surface (solid), the barotropic kinetic energy (dash-dot), and the variance of potential temperature at the top surface (dashed). (b) Kinetic energy spectra at different height values for the same run.

intensified, the surface modes likely have a more significant impact on the full flow.

The proposed model is, of course, still incomplete. In particular it produces insufficient potential energy near the surface at large scales- the GASP data shows potential and kinetic energy with nearly identical spectra at large and small scales, whereas the truncated model produces a weak APE spectrum at large scales. This is likely the result of our severe truncation of vertical modes. Observations of the atmospheric energy spectra at midtropospheric depths are sparse, but those that do exist show a spectral slope of kinetic energy a little steeper than -2 (Gao and Meriwether 1998). The model proposed here, by contrast, produces an interior (middepth) spectrum with a slope approaching -3 . The model is also free of divergent modes, which may play a role in the energy spectrum at some scale, although observations suggest that vorticity dominates divergence at least down to 100 km (Lindborg 2007).

Simple extensions to the model could yield more accurate results. For example, we assumed an infinite jump in stratification at the tropopause with no motion in the stratosphere. A model with a finite stratification jump at the tropopause and a free stratosphere could be derived following Juckes (1994). The applicability of $z$-coordinate simulations, using a high-vertical resolution model, is addressed in Tulloch and Smith (2009).

Acknowledgments. We thank the editor, K.-K. Tung, and two anonymous reviewers for constructive feedback that helped to clarify the manuscript. We also gratefully acknowledge helpful conversations with Glenn Flierl, Kevin Hamilton, Guillaume Lapeyre, and early support from Andrew Majda. This work was supported by NSF Grant OCE-0620874.

\section{APPENDIX A}

\section{PV Inversion Using a Green's Function}

\section{a. Green's function for the dynamic fields}

The streamfunction $\psi$ can be determined by inverting the linear elliptic problem in (2), subject to Neumann boundary conditions. This can be done in three ways: 1 ) by splitting the streamfunction into surface and interior components, as we have done in (4);2) by augmenting the potential vorticity with "delta sheets" at each surface (Bretherton 1966) and replacing the inhomogeneous boundary conditions in (2) with homogeneous ones; or 3) by using a Green's function method. Here we show, using the Green's function, that all three are equivalent.

Working in the spectral domain, defining $\mathcal{L} \hat{\psi} \equiv$ $\left(s d^{2} / d z^{2}-K^{2}\right) \hat{\psi}$, where $s=f^{2} / N^{2}$ and suppressing the dependence on $t,(2)$ can be expressed as

$$
\mathcal{L} \hat{\psi}=\hat{q}, \quad \hat{\psi}_{z}(H)=\hat{\theta}^{t}, \quad \text { and } \quad \hat{\psi}_{z}(0)=\hat{\theta}^{b}
$$

and its associated Green's function $g(z, \xi)$ therefore satisfies

$$
\mathcal{L} g(z, \xi)=\delta(z-\xi) \quad \text { and } \quad g_{z}(0, \xi)=g_{z}(H, \xi)=0
$$

Computing $\int_{0}^{H} g(z, \xi) \mathcal{L} \hat{\psi}(z)-\hat{\psi} \mathcal{L} g(z, \xi) d z$ yields the solution

$$
\hat{\psi}(z)=\int_{0}^{H} g(z, \xi) \hat{q}(\xi) d \xi+s g(z, 0) \hat{\theta}^{b}-s g(z, H) \hat{\theta}^{t} .
$$


By solving the homogeneous problem for $\hat{\psi}$ separately that $g$ is continuous and satisfies a jump condition), one on the domains $0 \leq z \leq \xi$ and $\xi \leq z \leq H$ (and assuming finds that

$$
g(\mu, z, \xi)= \begin{cases}-\frac{N^{2} H}{f^{2} \mu} \cosh \left(\mu \frac{\xi-H}{H}\right) \cosh \left(\mu \frac{z}{H}\right) \operatorname{csch} \mu \quad \text { for } \quad z \in(0, \xi) \\ -\frac{N^{2} H}{f^{2} \mu} \cosh \left(\mu \frac{z-H}{H}\right) \cosh \left(\mu \frac{\xi}{H}\right) \operatorname{csch} \mu \quad \text { for } \quad z \in(\xi, H)\end{cases}
$$

is the Green's function for the dynamic streamfunction $\hat{\psi}$.

In our formulation, using the decomposition of the streamfunction in (4), the integral in (A1) is $\hat{\psi}^{I}$ and the boundary terms are $\hat{\psi}^{b}$ and $\hat{\psi}^{t}$, respectively [this can be readily verified by comparing $\hat{\psi}$ obtained using Eq. (A2) with the $\hat{\psi}$ obtained by using the surface and interior solutions given in section 2].

Bretherton (1966) defined a modified PV:

$$
\tilde{q}=\hat{q}+s \delta(z) \hat{\theta}^{b}-s \delta(z-H) \hat{\theta}^{t},
$$

so that, with the modified PV, the streamfunction $\hat{\psi}$ solves

$$
\mathcal{L} \hat{\psi}=\tilde{q}, \quad \hat{\psi}_{z}(H)=\hat{\psi}_{z}(0)=0 .
$$

But this is equivalent to (A1), since

$$
\begin{aligned}
\int_{0}^{H} g(z, \xi) \tilde{q} d \xi= & \int_{0}^{H} g(z, \xi) \tilde{q}(\xi) d \xi+s g(z, 0) \hat{\theta}^{b} \\
& -s g(z, H) \hat{\theta}^{t} .
\end{aligned}
$$

[In fact, $\tilde{q}$ is simply the standardizing function (see Butkovskii 1982) for the boundary value problem in
(2).] Therefore, all three methods are equivalent. The advantage of using the streamfunction decomposition in (4) is that, among all three methods, this one allows the most straightforward, unambiguous numerical implementation, and avoids the need for highresolution finite-difference methods to capture surface effects.

\section{b. Green's function for the mean fields}

The mean velocity $U(z)$ must solve

$$
\begin{aligned}
\Gamma U \equiv s \frac{d^{2} U}{d z^{2}}=\beta-Q_{y}, \quad U_{z}(H) & =-\Theta_{y}^{t}, \quad \text { and } \\
U_{z}(0) & =-\Theta_{y}^{b},
\end{aligned}
$$

and so we seek a Green's function $G(z, \xi)$ that satisfies

$$
\Gamma U(z, \xi)=\delta(z-\xi) \quad \text { and } \quad G_{z}(H, \xi)=G_{z}(0, \xi)=0 .
$$

There is a function $G(z, \xi)$ that satisfies this problem, but is not a standard Green's function. The generalized Green's function

$$
\mathcal{G}(z, \xi)= \begin{cases}-\frac{N^{2} H}{f^{2}}\left\{\frac{1}{2}(z / H)^{2}+\frac{1}{2}[1-(\xi / H)]^{2}-\frac{1}{6}\right\} \text { for } \quad z \in(0, \xi), \\ -\frac{N^{2} H}{f^{2}}\left\{\frac{1}{2}(\xi / H)^{2}+\frac{1}{2}[1-(z / H)]^{2}-\frac{1}{6}\right\} \text { for } \quad z \in(\xi, H)\end{cases}
$$

yields a solution for $U(z)$ that is augmented by an arbitrary constant, $C$ :

$$
\begin{aligned}
U(z) & =\int_{0}^{H} \mathcal{G}(z, \xi)\left[\beta-Q_{y}(\xi)\right] d \xi+s \mathcal{G}(z, H) \Theta_{y}^{t}-s \mathcal{G}(z, 0) \Theta_{y}^{b}+C \\
& =\int_{0}^{H} \mathcal{G}(z, \xi) w(\xi) d \xi+C
\end{aligned}
$$

where $w$ is the standardizing function

$$
w(z)=\beta-Q_{y}(z)+s \Theta_{y}^{b} \delta(z)-s \Theta_{y}^{t} \delta(z-H),
$$


which must satisfy $\int_{0}^{H} w(z) d z=0$ (Butkovskii 1982, p. 30). Using the expansion $U(z)=U^{\mathrm{bc}} \phi(z)+U^{S}(z)$, and Eqs. (8) and (A3) become

$$
\beta-Q_{y}(z)=-\lambda^{2} U^{\mathrm{bc}} \phi(z)+\Gamma U^{S} .
$$

Using this expression in (A4), a few lines of computation reveals that $-\int_{0}^{H} \mathcal{G}(z, \xi) \Gamma U^{I} d \xi=-U^{\mathrm{bc}} \int_{0}^{H} \mathcal{G}(z$, $\xi) \Gamma \phi(\xi) d \xi=U^{\mathrm{bc}} \phi(z)$, as it should, and

$$
\begin{aligned}
U^{S}(z)= & \int_{0}^{H} \mathcal{G}(z, \xi) \Gamma U^{S} d \xi+s \mathcal{G}(z, H) \Theta_{y}^{t} \\
& -s \mathcal{G}(z, 0) \Theta_{y}^{b}+C .
\end{aligned}
$$

Demanding that $\Gamma U^{S}=$ const, $\langle w\rangle=0$ and $\left\langle U^{S}\right\rangle=0$ then yields $C=0$ and the form stated in (16) follows [note that $\left.\int_{0}^{H} G(z, \xi) d \xi=0\right]$.

\section{APPENDIX B}

\section{Details of the TMTS Equations}

In forming the barotropic and baroclinic model in Eqs. (18c) and (18d), interaction coefficients between the vertical structure functions $\phi$ arise. Using the expressions for $\phi^{t, b}$ in (11) we have

$$
\left\langle\phi^{t}\right\rangle=\left\langle\phi^{b}\right\rangle=\frac{1}{H} \int_{0}^{H} \frac{\cosh (\mu z / H)}{\cosh \mu} d z=\mu^{-1} \tanh \mu .
$$

Notice that because the surface modes have both vertical and horizontal dependence, the interaction terms involving surface functions are functions of $\mu$. We can form the other interaction terms using $\phi(z)$ from (9), and computing the integrals:

$$
\begin{gathered}
\left\langle\phi \phi^{t}\right\rangle=-\left\langle\phi \phi^{b}\right\rangle=\frac{1}{H} \int_{0}^{H} \sqrt{2} \cos (\pi z / H) \frac{\cosh (\mu z / H)}{\cosh \mu} d z=-\frac{\sqrt{2} \mu}{\mu^{2}+\pi^{2}} \tanh \mu \\
\left\langle\phi \phi \phi^{t}\right\rangle=\left\langle\phi \phi \phi^{b}\right\rangle=\frac{1}{H} \int_{0}^{H} 2 \cos ^{2}(\pi z / H) \frac{\cosh (\mu z / H)}{\cosh \mu} d z=\frac{2}{\mu} \frac{\mu^{2}+2 \pi^{2}}{\mu^{2}+4 \pi^{2}} \tanh \mu
\end{gathered}
$$

Since $\hat{\psi}(z)=\hat{\psi}^{\mathrm{bt}}+\phi(z) \hat{\psi}^{\mathrm{bc}}+\phi^{t}(z) \hat{\psi}^{t}+\phi^{b}(z) \hat{\psi}^{b}$ the barotropic projection is simply the vertical average

$$
\langle\hat{\psi}\rangle=\hat{\psi}^{\mathrm{bt}}+\left\langle\phi^{t}\right\rangle \hat{\psi}^{t}+\left\langle\phi^{b}\right\rangle \hat{\psi}^{b},
$$

where the baroclinic term has vanished since $\langle\phi\rangle=0$. Summarizing, the projections of the total streamfunction onto various internal modes are

$$
\begin{aligned}
& \langle\hat{\psi}\rangle=\hat{\psi}^{\mathrm{bt}}+\gamma_{0}\left(\hat{\psi}^{t}+\hat{\psi}^{b}\right) \text {, where } \gamma_{0} \equiv \mu^{-1} \tanh \mu, \\
& \langle\phi \hat{\psi}\rangle=\hat{\psi}^{\mathrm{bc}}+\gamma_{1}\left(\hat{\psi}^{t}+\hat{\psi}^{b}\right) \text {, where } \gamma_{1} \equiv \frac{\sqrt{2} \mu}{\mu^{2}+\pi^{2}} \tanh \mu, \quad \text { and } \\
& \langle\phi \phi \hat{\psi}\rangle=\hat{\psi}^{\mathrm{bt}}+\gamma_{2}\left(\hat{\psi}^{t}+\hat{\psi}^{b}\right), \text { where } \gamma_{2} \equiv \frac{2}{\mu} \frac{\mu^{2}+2 \pi^{2}}{\mu^{2}+4 \pi^{2}} \tanh \mu .
\end{aligned}
$$

We must also compute interactions between the mean shear components and vertical mode structures. Interactions between the baroclinic mode $\phi$ and the surface shear (16) are given by

$$
\begin{aligned}
\left\langle\phi U^{S}\right\rangle & =\frac{1}{H} \int_{0}^{H} \sqrt{2} \cos (\pi z / H) U^{S}(z) d z \\
& =\frac{\sqrt{2} H}{\pi^{2}}\left(\Theta_{y}^{t}+\Theta_{y}^{b}\right),
\end{aligned}
$$

$$
\left\langle\phi \phi U^{S}\right\rangle=\frac{1}{H} \int_{0}^{H} 2 \cos ^{2}(\pi z / H) U^{S}(z) d z=-\frac{H}{4 \pi^{2}}\left(\Theta_{y}^{t}-\Theta_{y}^{b}\right) .
$$

The projections of the mean velocity that appear in (18) are then

$$
\begin{aligned}
\langle\phi U\rangle & =U^{\mathrm{bc}}+\frac{\sqrt{2} H}{\pi^{2}}\left(\Theta_{y}^{t}+\Theta_{y}^{b}\right), \\
\langle\phi \phi U\rangle & =-\frac{H}{4 \pi^{2}}\left(\Theta_{y}^{t}-\Theta_{y}^{b}\right) .
\end{aligned}
$$




\section{APPENDIX C}

\section{Linear Equations}

Upon neglecting the nonlinear terms in (18), assuming a wave solution

$$
\left(\hat{\psi}^{t}, \hat{\psi}^{b}, \hat{\psi}^{\mathrm{bt}}, \hat{\psi}^{\mathrm{bc}}\right)=\Re\left(\hat{\varphi}^{t}, \hat{\varphi}^{b}, \hat{\varphi}^{\mathrm{bt}}, \hat{\varphi}^{\mathrm{bc}}\right) \mathrm{e}^{-i \omega t},
$$

and considering only zonal wave instabilities $(\ell=0)$, so that the phase speed is $c=\omega / k$, we obtain

$$
\begin{aligned}
c \hat{\varphi}^{t}= & U(H) \hat{\varphi}^{t}+H \Theta_{y}^{t}(\mu \tanh \mu)^{-1} \\
& \times\left(\hat{\varphi}^{\mathrm{bt}}-\sqrt{2} \hat{\varphi}^{\mathrm{bc}}+\hat{\varphi}^{t}+\hat{\varphi}^{b} \operatorname{sech} \mu\right), \\
c \hat{\varphi}^{b}= & U(0) \hat{\varphi}^{b}-H\left(\Theta_{y}^{b}+i r \frac{K^{2}}{k}\right)(\mu \tanh \mu)^{-1} \\
& \times\left(\hat{\varphi}^{\mathrm{bt}}+\sqrt{2} \hat{\varphi}^{\mathrm{bc}}+\hat{\varphi}^{t} \sec h \mu+\hat{\varphi}^{b}\right), \\
c \hat{\varphi}^{\mathrm{bt}}= & \langle\phi U\rangle \mu^{-2}\left(\mu^{2}+\pi^{2}\right) \hat{\varphi}^{\mathrm{bc}}-\left(\beta-\Gamma U^{S}\right) \\
& \times K^{-2}\left[\hat{\varphi}^{\mathrm{bt}}+\gamma_{0}\left(\hat{\varphi}^{t}+\hat{\varphi}^{b}\right)\right] \\
& -U^{\mathrm{bc}} \lambda^{2} K^{-2}\left[\hat{\varphi}^{\mathrm{bc}}-\gamma_{1}\left(\hat{\varphi}^{t}-\hat{\varphi}^{b}\right)\right] \\
c \hat{\varphi}^{\mathrm{bc}}= & \langle\phi U\rangle \mu^{2}\left(\mu^{2}+\pi^{2}\right)^{-1} \hat{\varphi}^{\mathrm{bt}}+\langle\phi \phi U\rangle \hat{\varphi}^{\mathrm{bc}} \\
& -\left(\beta-\Gamma U^{S}\right) K^{-2} \mu^{2}\left(\mu^{2}+\pi^{2}\right)^{-1} \\
& \times\left[\hat{\varphi}^{\mathrm{bc}}-\gamma_{1}\left(\hat{\varphi}^{t}-\hat{\varphi}^{b}\right)\right]-U^{\mathrm{bc}} \lambda^{2} K^{-2} \mu^{2}\left(\mu^{2}+\pi^{2}\right)^{-1} \\
& \times\left[\hat{\varphi}^{\mathrm{bt}}+\gamma_{2}\left(\hat{\varphi}^{t}+\hat{\varphi}^{b}\right)\right],
\end{aligned}
$$

which is a $4 \times 4$ eigenvalue problem with $\left(\hat{\varphi}^{t}, \hat{\varphi}^{b}, \hat{\varphi}^{\mathrm{bt}}\right.$, $\left.\hat{\varphi}^{\mathrm{bc}}\right)$ as the eigenvector and the phase speed $c$ as the eigenvalue. The largest imaginary part of $\omega=c k$ is then plotted in Figs. 2 and 3.

\section{REFERENCES}

Blumen, W., 1978: Uniform potential vorticity flow. Part I: Theory of wave interactions and two-dimensional turbulence. J. Atmos. Sci., 35, 774-783.

Bretherton, F. P., 1966: Critical layer instability in baroclinic flows. Quart. J. Roy. Meteor. Soc., 92, 325-334.

Butkovskii, A. G., 1982: Green's Functions and Transfer Functions Handbook. Halsted Press, 236 pp.

Charney, J. G., 1947: Dynamics of long waves in a baroclinic westerly current. J. Meteor., 4, 136-162.

_ , 1971: Geostrophic turbulence. J. Atmos. Sci., 28, 1087-1095. , and M. E. Stern, 1962: On the stability of internal baroclinic jets in a rotating atmosphere. J. Atmos. Sci., 19, 159-172.

Cho, J. Y. N., and E. Lindborg, 2001: Horizontal velocity structure functions in the upper troposphere and lower stratosphere 1. Observations. J. Geophys. Res., 106, 10 223-10 232.

— , R. E. Newell, and J. D. Barrick, 1999: Horizontal wavenumber spectra of winds, temperature, and trace gases during the Pacific exploratory missions. Part II: Gravity waves, quasitwo-dimensional turbulence, and vertical modes. J. Geophys. Res., 104, 16 297-16 308

Davies, H. C., and C. H. Bishop, 1994: Eady edge waves and rapid development. J. Atmos. Sci., 51, 1930-1946.

Dewan, E. M., 1979: Stratospheric wave spectra resembling turbulence. Science, 204, 832-835.

_ 1997: Saturated-cascade similitude theory of gravity wave spectra. J. Geophys. Res., 102, 29 799-29 817.

Eady, E. T., 1949: Long waves and cyclone waves. Tellus, 1, $33-52$.

Fox-Rabinovitz, M., and R. S. Lindzen, 1993: Numerical experiments on consistent horizontal and vertical resolution for atmospheric models and observing systems. Mon. Wea. Rev., 121, 264-271.

Gao, X., and J. W. Meriwether, 1998: Mesoscale spectral analysis of in situ horizontal and vertical wind measurements at $6 \mathrm{~km}$. J. Geophys. Res., 103, 6397-6404.

Gkioulekas, E., and K. Tung, 2007a: Is the subdominant part of the energy spectrum due to downscale energy cascade hidden in quasi-geostrophic turbulence? Discrete Contin. Dyn. Syst. Ser. B., 7, 293-314.

- and - 2007b: A new proof on net upscale energy cascade in 2D and QG turbulence. J. Fluid Mech., 576, 173-189.

Green, J. S. A., 1960: A problem in baroclinic instability. Quart. J. Roy. Meteor. Soc., 86, 237-251.

Hamilton, K., Y. O. Takahashi, and W. Ohfuchi, 2008: Mesoscale spectrum of atmospheric motions investigated in a very fine resolution global general circulation model. J. Geophys. Res., 113, D18110, doi:10.1029/2008JD009785.

Heifetz, E., J. Methven, B. J. Hoskins, and C. H. Bishop, 2004: The counter-propagating Rossby-wave perspective on baroclinic instability. II: Application to the Charney model. Quart. J. Roy. Meteor. Soc., 130, 233-258.

Held, I. M., R. T. Pierrehumbert, S. T. Garner, and K. L. Swanson, 1995: Surface quasigeostrophic dynamics. J. Fluid Mech., 282, $1-20$.

Holopainen, E., and J. Kaurola, 1991: Decomposing the atmospheric flow using potential vorticity framework. J. Atmos. Sci., 48, 2614-2625.

Hoskins, B. J., and F. P. Bretherton, 1972: Atmospheric frontogenesis models: Mathematical formulation and solution. J. Atmos. Sci., 29, 11-37.

—, M. E. McIntyre, and A. W. Robertson, 1985: On the use and significance of isentropic potential vorticity maps. Quart. J. Roy. Meteor. Soc., 111, 877-946.

Juckes, M. N., 1994: Quasigeostrophic dynamics of the tropopause. J. Atmos. Sci., 51, 2756-2768.

Kitamura, Y., and Y. Matsuda, 2006: The $k_{h}^{-3}$ and $k_{h}^{-5 / 3}$ energy spectra in stratified turbulence. Geophys. Res. Lett., 33, L05809, doi:10.1029/2005GL024996.

Koshyk, J. N., and K. P. Hamilton, 2001: The horizontal kinetic energy spectrum and spectral budget simulated by a highresolution troposphere-stratosphere-mesosphere GCM. J. Atmos. Sci., 58, 329-348.

,$- \ldots$, and J. D. Mahlman, 1999: Simulation of $k^{-5 / 3}$ mesoscale spectral regime in the GFDL SKYHI general circulation model. Geophys. Res. Lett., 26, 843-846.

Lapeyre, G., and P. Klein, 2006: Dynamics of the upper oceanic layers in terms of surface quasigeostrophy theory. J. Phys. Oceanogr., 36, 165-176.

Lilly, D. K., 1989: Two-dimensional turbulence generated by energy sources at two scales. J. Atmos. Sci., 46, 2026-2030. 
Lindborg, E., 1999: Can the atmospheric kinetic energy spectrum be explained by two-dimensional turbulence? J. Fluid Mech., 388, 259-288.

, 2005: The effect of rotation on the mesoscale energy cascade in the free atmosphere. Geophys. Res. Lett., 32, L01809, doi:10.1029/2004GL021319.

_ - 2007: Horizontal wavenumber spectra of vertical vorticity and horizontal divergence in the upper troposphere and lower stratosphere. J. Atmos. Sci., 64, 1017-1025.

Lindzen, R., 1994: The Eady problem with zero PV gradient but beta unequal to zero. J. Atmos. Sci., 51, 3221-3226.

McWilliams, J. C., and J. H. S. Chow, 1981: Equilibrium geostrophic turbulence. Part I: A reference solution in a $\beta$-plane channel. J. Phys. Oceanogr., 11, 921-949.

Nastrom, G. D., and K. S. Gage, 1985: A climatology of atmospheric wavenumber spectra of wind and temperature observed by comercial aircraft. J. Atmos. Sci., 42, 950-960.

Pedlosky, J., 1964: The stability of currents in the atmosphere and ocean. Part I. J. Atmos. Sci., 21, 201-219.

- 1987: Geophysical Fluid Dynamics. 2nd ed. Springer, 710 pp.

Phillips, N. A., 1954: Energy transformations and meridional circulations associated with simple baroclinic waves in a twolevel, quasi-geostrophic model. Tellus, 6, 273-286.

Skamarock, W. C., 2004: Evaluating mesoscale NWP models using kinetic energy spectra. Mon. Wea. Rev., 132, 3019-3032.

—, and J. B. Klemp, 2008: A time-split nonhydrostatic atmospheric model for weather research and forecasting applications. J. Comput. Phys., 227, 3465-3485.
Smith, K. S., G. Boccaletti, C. C. Henning, I. N. Marinov, C. Y. Tam, I. M. Held, and G. K. Vallis, 2002: Turbulent diffusion in the geostrophic inverse cascade. J. Fluid Mech., 469, 13-48.

Snyder, C., T. M. Hamill, and S. B. Trier, 2003: Linear evolution of error covariances in a quasigeostrophic model. Mon. Wea. Rev., 131, 189-205.

Solomon, A., and R. S. Lindzen, 2000: The impact of resolution on a numerical simulation of the barotropic point jet. J. Atmos. Sci., 57, 3799-3816.

Takahashi, Y., K. Hamilton, and W. Ohfuchi, 2006: Explicit global simulation of the mesoscale spectrum of atmospheric motions. Geophys. Res. Lett., 33, L12812, doi:10.1029/2006GL026429.

Tulloch, R. T., and K. S. Smith, 2006: A theory for the atmospheric energy spectrum: Depth-limited temperature anomalies at the tropopause. Proc. Natl. Acad. Sci. USA, 103, 14 690-14 694.

— and - 2009: On the numerical representation of surface dynamics in quasigeopstrophic turbulence: Application to the nonlinear Eady model. J. Atmos. Sci., in press.

Tung, K. K., and W. W. Orlando, 2003a: The $k^{-3}$ and $k^{-5 / 3}$ energy spectrum of atmospheric turbulence: Quasigeostrophic twolevel model simulation. J. Atmos. Sci., 60, 824-835.

$\longrightarrow$, and - 2003b: On the differences between 2D and QG turbulence. Discrete Contin. Dyn. Syst. Ser. B, 3, 145-162.

Vallis, G. K., G. J. Shutts, and M. E. Gray, 1997: Balanced mesoscale motion and stratified turbulence forced by convection. Quart. J. Roy. Meteor. Soc., 123, 1621-1652.

VanZandt, T. E., 1982: A universal spectrum of buoyancy waves in the atmosphere. Geophys. Res. Lett., 9, 575-578. 